Review

\title{
Strengths, Weaknesses, Opportunities and Threats: Computational Studies of Mn- and Fe-Catalyzed Epoxidations
}

\author{
Filipe Teixeira * and M. Natália D. S. Cordeiro * \\ LAQV-REQUIMTE, Departamento de Química e Bioquímica, Faculdade de Ciências, Universidade do Porto, \\ Rua do Campo Alegre, 4169-007 Porto, Portugal \\ * Correspondence: filipe.teixeira@fc.up.pt (F.T.); ncordeir@fc.up.pt (M.N.D.S.C.); \\ Tel.: +351-220-402-552 (F.T.); +351-220-402-502 (M.N.D.S.C.)
}

Academic Editor: José R. B. Gomes

Received: 4 November 2016; Accepted: 20 December 2016; Published: 23 December 2016

\begin{abstract}
The importance of epoxides as synthetic intermediates in a number of highly added-value chemicals, as well as the search for novel and more sustainable chemical processes have brought considerable attention to the catalytic activity of manganese and iron complexes towards the epoxidation of alkenes using non-toxic terminal oxidants. Particular attention has been given to $\mathrm{Mn}$ (salen) and $\mathrm{Fe}$ (porphyrin) catalysts. While the former attain remarkable enantioselectivity towards the epoxidation of cis-alkenes, the latter also serve as an important model for the behavior of cytochrome P450, thus allowing the exploration of complex biological processes. In this review, a systematic survey of the bibliographical data for the theoretical studies on $\mathrm{Mn}$ - and Fe-catalyzed epoxidations is presented. The most interesting patterns and trends are reported and finally analyzed using an evaluation framework similar to the SWOT (Strengths, Weaknesses, Opportunities and Threats) analysis performed in enterprise media, with the ultimate aim to provide an overview of current trends and areas for future exploration.
\end{abstract}

Keywords: theoretical modeling; density functional theory; reaction mechanism; iron; manganese

\section{Introduction and Context}

Epoxides are cyclic ethers in which one oxygen atom and two carbon atoms are arranged approximately as an equilateral triangle. A significant amount of strain energy emerges from this particular arrangement of atoms, making any ring-opening reaction highly exothermic. These highly reactive, yet apparently simple structures may take part in a number of different reactions [1]. Moreover, up to two chiral carbon atoms may form upon ring opening. Because of this, epoxides are well-established intermediates in asymmetric synthesis, being important intermediates in the synthesis of more complex chemical structures, such as medicines, fragrances and polymers [2,3]. Different synthetic routes are available for the formation of epoxides, usually involving the use of a organic epoxidizing agents, such as organic peracids (or peroxy acids), oxone (potassium peroxymonosulfate), dioxiranes or oxaziridines. However, the use of organic epoxidizing agents is hindered by the high cost of these compounds, their relatively low active oxygen content (worst atom economy) and their potentially harmful by-products. Peroxides, in particular hydrogen peroxide, $\mathrm{H}_{2} \mathrm{O}_{2}$ and tert-butyl hydroperoxide (TBHP), offer viable alternatives to the use of molecular oxygen. As an oxidant, $\mathrm{H}_{2} \mathrm{O}_{2}$ offers the largest atom economy (besides molecular $\mathrm{O}_{2}$ ). Being liquids, both $\mathrm{H}_{2} \mathrm{O}_{2}$ and TBHP are easier to transport, store and manipulate than $\mathrm{O}_{2}$. Their by-products are either environmentally harmless (water) or can be recycled into the production of TBHP [4]. Moreover, $\mathrm{H}_{2} \mathrm{O}_{2}$ /organic mixtures are much safer to handle than those with $\mathrm{O}_{2}$, and TBHP/organic mixtures are even more stable. What 
is more, the use of efficient catalysts, such as zeolites, metal oxides and transition metal complexes, allows for milder reaction conditions [5], allowing the synthesis of structurally-complex epoxides.

Transition Metal (TM) complexes are very important catalysts in the epoxidation of simple alkenes and other olefinic materials, such as allylic alcohols using $\mathrm{H}_{2} \mathrm{O}_{2}$ or TBHP as primary oxygen sources. Their use allows the synthesis of more complex epoxides, as well as the regioselective epoxidation of poly-unsaturated compounds or the stereoselective formation of chiral epoxides. The field of alkene epoxidation catalyzed by transition metal complexes is remarkably diverse, with a multitude of metal/ligand combinations being available [6]. What is more, some of the most successful approaches for achieving high stereoselectivity in the epoxidation of unfunctionalized olefins evolved from a biomimetic strategy. Cytochrome P-450 enzymes are a family of porphyrin-bearing proteins involved in xenobiotic metabolism, biosynthesis of steroids, lipids, vitamins and other natural products [7]. Synthetic iron and manganese bearing metalloporphyrins (1, in Figure 1) were originally developed in the 1980s as models for investigating the reactivity of P-450 [8] . Many of these compounds have been reported to catalyze the epoxidation of unfunctionalized olefins.

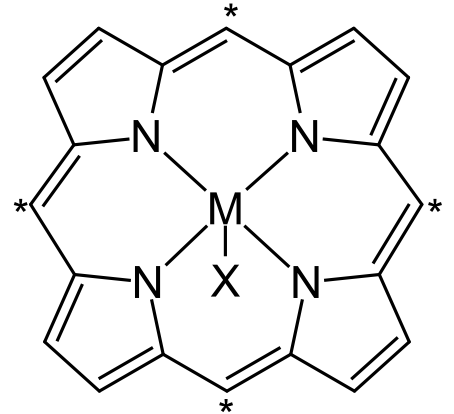

(1)

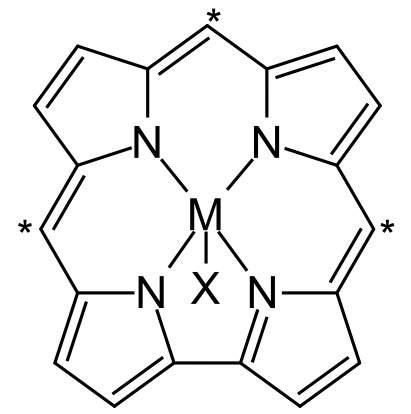

(3)

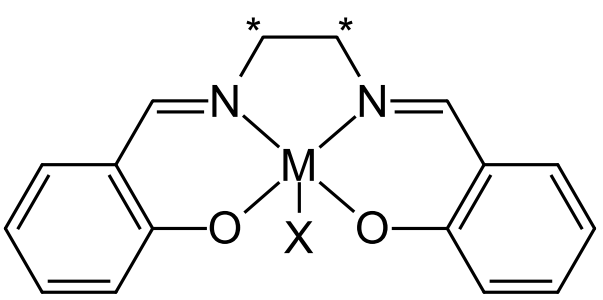

(2)

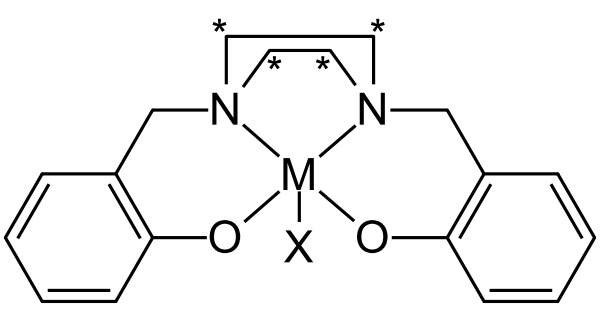

(4)

Figure 1. General structures of metalloporphyrin (1), metallosalen (2), metallocorrole (3) and metallo-bis-amino-phenolate (4) complexes, in which $\mathrm{M}=\mathrm{Mn}$, Fe. Positions and/or groups marked with an asterisk ${ }^{*}$ ) are usually responsible for providing chirality to the complex, via the introduction of chiral substituents.

At the same time, the $\mathrm{N}, \mathrm{N}$-ethylene bis(salicyldeneaminato) ligand ( 2 in Figure 1 ; henceforward salen) aroused the interest of synthetic chemists as an alternative model compound for the active site of P-450 [9]. Many of these complexes bear catalytic activity towards the epoxidation of unfunctionalized olefins, and over 2500 transition metal complexes based on the salen scaffold have been described and characterized [10]. Chromium-salen, nickel-salen and manganese-salen complexes bear some interesting catalytic activity towards the epoxidation of olefins [11]. However, manganese-salen (Mn(salen)) complexes are by far the most efficient catalysts carrying the salen ligand [9]. This led to the establishment of the Mn(salen) catalyzed epoxidation of olefins, also known as the Jacobsen-Katsuki epoxidation, to become one of the most important preparative epoxidation techniques [11]. Other 
relevant ligand features in catalysts for alkene epoxidation are corroles (3, structurally similar to porphyrins) [12] and bis-amino-phenolate ligands (4, structurally related to salen) [13], which are also represented in Figure 1.

The prominence of porphyrin and salen scaffolds in the design of $\mathrm{Mn}$ and Fe catalysts for epoxidation reactions is well illustrated by some past reviews on the matter. In particular, iron-porphyrins have been the subject of extensive reviews, due to their biological relevance as part of cytochrome P450 [14,15], with their use as catalysts in epoxidation reactions being highlighted on many occasions [16]. On the other hand, the efficiency of the Jacobsen-Katsuki epoxidation prompted the study of $\mathrm{Mn}$ (salen), which is much more focused on their catalytic activity towards the epoxidation of alkenes.

Historically, two works stand in this matter: the comprehensive survey of the variants on the Jacobsen-Katsuki process made by Katsuki in 1996 [9] and the extended review by McGarrigle and Gilheany [11] on chromium and manganese-salen-promoted epoxidation of alkenes. The most interesting aspect of McGarrigle and Gilheany's review is their account of the new contributions provided by theoretical chemistry studies and also the main shortcomings in such studies. According to McGarrigle and Gilheany [11], the three main themes in theoretical studies are: the geometry, spin state and radical nature of the active catalyst; the conformation of both the catalyst in both active and inactive forms; and the reaction mechanism. This latter category can be further sub-divided into studying the formation of the active specie(s) responsible for the epoxidation of the substrate and the oxidation of the $\mathrm{C}=\mathrm{C}$ bond per se. What is more, the main shortcomings on the theoretical study of $\mathrm{Mn}$ (salen) systems were also identified. These include the choice of computational method: whether it is Hartree-Fock (HF), Density Functional Theory (DFT) or post-HF methods, such as Complete Active-Space Self Consistent Field (CASSCF), Coupled Cluster (CC) or Møller-Plesset Perturbation Theory (MP2). In the case of DFT calculations, a further issue lies with the choice of a particular functional, with different functionals giving qualitatively different results $[11,17,18]$. Also associated with the choice of computational method comes the choice of the basis set on which the resulting wavefunction (density function in DFT calculations) is expressed. Furthermore, one of the most prominent critiques made by McGarrigle and Gilheany [11] lies in the use of truncated models, namely Mn(acacen) (5, in Figure 2), which does not carry the chiral nature of the more elaborated Jacobsen catalyst (6, in Figure 2), nor represent the conjugated $\pi$ system of the simpler salen complexes (2). As stated by McGarrigle and Gilheany [11], such shortcomings are mostly due to the computational cost of ab initio calculations on this type of system, which should be surpassed with the development of computer technology and computational methods.

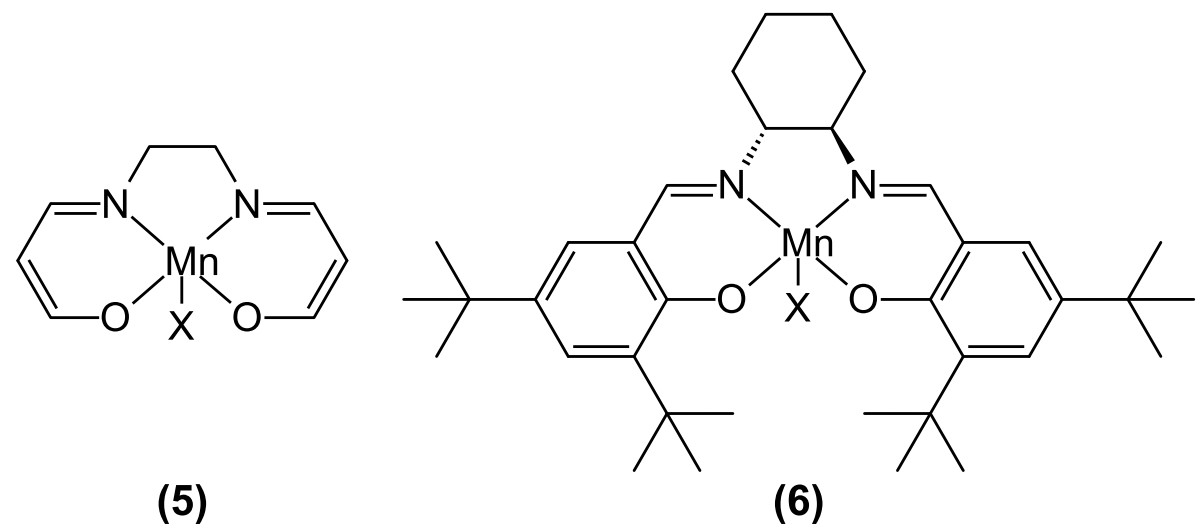

Figure 2. General formulae of Mn(acacen) (5); and the Jacobsen catalyst (6). 
Undoubtedly, many of the insights provided by McGarrigle and Gilheany [11] also apply to the theoretical study of other manganese and iron catalysts. It is thus justified that, over a decade later, the current state of the art in the theoretical modeling of $\mathrm{Mn}$ - and Fe-catalyzed epoxidations is addressed.

\subsection{Scope of the Review}

This review covers the contributions made to the understanding of the manganese and iron-catalyzed epoxidation of alkenes and other olefinic substrates during the last 20 years (1996-2016). We furthermore endeavor to interpret the information contained in these works in terms of a Strengths, Weaknesses, Opportunities and Threats (SWOT) analysis. For this aim, the primary bibliographic sources were collected from Thomson Reuters' ISI Web of Science matching the following search criteria: "(MANGANESE OR MN OR IRON OR FE) AND EPOXIDATION AND (THEORETICAL OR COMPUTATIONAL AB INITIO OR FIRST PRINCIPLES OR DFT OR MP2 OR CASSCF)", which was further filtered to match the time frame mentioned above.

A total of 210 entries were collected (including one errata). Four entries were Abstracts Papers of the American Chemical Society and were excluded from further consideration. Moreover, three entries [19-21] were not written in English (apart from the abstract), thus limiting our access to the information therein. Further inspection of the abstracts led to discarding 92 sources due to a lack of theoretical content, or a focus on other reactions (mainly $\mathrm{C}-\mathrm{H}$ hydroxylation), or a focus on other TM, such as chromium, ruthenium, titanium and vanadium. This bibliographical survey was carried out in August 2016, but the release of bibliographic sources matching these search criteria was monitored until October 2016. Statistical analysis and data mining of the bibliographical data were performed using R [22].

\section{Statistical Meta-Analysis}

A collection of the most common words found in the abstracts of the bibliographical sources (after removing common English stop words) highlights the importance of DFT methods in this research area, with the B3LYP functional being often mentioned. Indeed, $106(90 \%)$ of the entries mentioned DFT calculations. Surprisingly, $18 \%$ of the entries also mentioned the HF method, although further inspection reveals that this is usually done either in the context of the exact exchange in DFT functionals or when obtaining the reference wavefunction for more advanced post-HF methods, such as MP2 (mentioned in $8.5 \%$ of the sources), coupled cluster (17\% of the entries mention CCSD) or CASSCF (also mentioned in 13\% of the sources). Other relevant terms pertaining to the level of theory used in these studies reveal some popularity of Valence Bond (VB) calculations, mentioned in 10 sources, and also classical Molecular Mechanics (MM), which is mentioned in 15 sources (all of which also mention hybrid QM/MM calculations).

Within the realm of DFT calculations, Table 1 shows almost the complete hegemony of B3LYP, which despite its inadequacy towards the treatment of TM systems [17,23], remains as the de facto standard for DFT calculations. Other common functionals are BP86and LSD. As can be inferred from Table 1, a significant number of papers cites results from more than one functional. It is well known that B3LYP and other hybrid functionals (containing exact HF exchange) are prone to overestimate the stability of high-spin complexes, whereas local functionals (such as BP86 and LSD) tend to do otherwise [23]. Thus, the use of more than one functional (especially different in their exchange component) might be a strategy to reach an ad hoc middle ground. This hypothesis will be addressed later in this work. From among the newer meta-GGAfunctionals, only the M06suite stands out. This might come as a surprise, since the M06, M06-HF and M06-2Xfunctionals are specially tailored for predicting TM thermodynamic properties [24]. On the other hand, their relative lower age and the fewer number of computational implementations available might cause the scarcity of studies using them. What is more, newer double hybrid functionals, such as B2PLYP(which contains a component of explicit MP2 correlation), are yet to be applied to the study of these complexes and their reactivity. 
This absence is possibly due to the high computational cost of calculating the explicit MP2 correlation component of B2PLYP.

Table 1. Density functionals most often mentioned in the bibliographic sources and the percentage within the sources referring to Density Functional Theory (DFT) calculations.

\begin{tabular}{ccr}
\hline Functional & $\boldsymbol{N}$ & \multicolumn{1}{c}{$\%$} \\
\hline B3LYP & 89 & 84.0 \\
BP86 & 27 & 25.5 \\
LDAor LSD & 15 & 14.2 \\
PW91 & 9 & 8.5 \\
BLYP & 8 & 7.5 \\
PBE & 8 & 7.5 \\
mPBE & 8 & 7.5 \\
OPBE & 7 & 6.6 \\
B3PW91 & 4 & 3.8 \\
PBE0 & 3 & 2.8 \\
M06 & 3 & 2.8 \\
B97 & 2 & 1.9 \\
PW91B95 & 2 & 1.9 \\
\hline
\end{tabular}

Our analysis of the most common terms in the abstracts of the bibliographical sources also gave some emphasis on terms often associated with the study of reaction mechanisms, such as "mechanism", "transfer", "intermediates", "catalytic" and "barrier". Another interesting group of words highlighted in our frequency analysis is strongly related to the questions surrounding the different spin multiplicities accessible in Mn and Fe complexes, including terms such as "triplet" and "doublet". Finally, a significant number of articles mentions the term "surface". Although this is mostly done in the context of probing the Potential Energy Surface (PES), a significant number of sources also contain the terms "immobilized" (12 entries) or "anchored" (seven entries), denoting the growing interest in the theoretical study of novel materials with catalytic activities in which these complexes are immobilized into a nanostructured matrix [25-27].

Regarding the ligands featured in the bibliographic sources, $63 \%$ of the sources mention porphyrin ligands (74 sources), while 44 mention the salen ligand (37\%). Corroles are less popular, being mentioned by only 11 sources, whereas "bisphenolate" ligands are mentioned in only 10 articles. A significant number of authors labels their ligands as being generically "non-heme". Such a term is found in about $20 \%$ of the sources. Interestingly, $81 \%$ of the sources mentions "iron", whereas "manganese" is found in $77 \%$ of the bibliography. This reflects the fact that a significant amount of work is done by comparing both metals or that they serve as mutual references when evaluating one's results.

Despite the 20-year time frame allowed for the bibliographic survey, 50\% of the sources are from 2010 or later. What is more, $25 \%$ of the sources date from 2014, 2015 and 2016. This reflects not only the growing interest of these reactions, but also the aforementioned development of computer technology making the first principles study of systems such as $\mathrm{Mn}$ (salen) and Fe(porphyrin) complexes accessible to many researchers [11].

Further insight into the sources shows that studies focusing on manganese-catalyzed epoxidations (particularly those involving $\mathrm{Mn}$ (salen) complexes) usually focus on the reaction mechanism. On the other hand, studies involving iron-porphyrin catalysts are usually directed towards an explanation of cytochrome P450's reactivity, including the effect of mutations in the protein moiety. This hinders the comparison between different theoretical studies. Because of this, our review will mainly focus on the theoretical insights into the $\mathrm{Mn}$ (salen)-catalyzed epoxidations, which will serve as a comparative standard to the other theoretical surveys. We shall also start by highlighting the main points made by McGarrigle and Gilheany [11] before moving on to a more critical assessment of the SWOTs facing this field of study. 


\section{Ground State Spin Multiplicity of Mn(Salen) Species}

Transition state metal complexes are well known for having low lying excited states, usually corresponding to different spin multiplicities. Thus, a common benchmark found in the surveyed studies is the determination of the ground state nature of the intervening species. Indeed, the determination of the ground state spin multiplicity of $\mathrm{Mn}$ (salen) and oxo-Mn(salen) complexes has attracted considerable attention and will serve as a case study for the difficulties that theoretical studies face.

Manganese(III) has a $3 d^{4}$ configuration. It has thus been suggested that the most stable electronic configuration of $\mathrm{Mn}$ (III) would involve having all four $d$ electrons unpaired, yielding a quintuplet state. However, the fact that the salen ligand assumes a slightly bent conformation [28] and the effect of axial coordination by a donor ligand may alter this outcome. A triplet (two unpaired $d$ electrons) or a singlet (no unpaired electrons) ground state may therefore be a viable hypotheses [29-31]. Table 2 shows the relative gas-phase energies of the lowest-energy singlet, triplet and quintuplet states of different non-oxo Mn(III) complexes, determined using different DFT functionals and basis sets.

Table 2. Relative gas-phase energies (in $\mathrm{kJ} \cdot \mathrm{mol}^{-1}$ ) of the singlet, triplet and quintet states of $\mathrm{Mn}$ complexes using different DFT methods. The ground state in each entry is highlighted in bold.

\begin{tabular}{|c|c|c|c|c|c|c|}
\hline Functional & Basis Set & Complex & Singlet & Triplet & Quintuplet & Reference \\
\hline BP86 & $\operatorname{DZVP}^{a}$ & $\mathrm{Mn}$ (acacen)Cl & +37.2 & 0.0 & +33.0 & [32] \\
\hline BP86 & $\mathrm{BS}^{b}$ & Mn(acacen $)^{+}$ & $\mathrm{NA}^{c}$ & +62.0 & 0.0 & [17] \\
\hline B3LYP & BS1 & $\operatorname{Mn}(\text { acacen })^{+}$ & NA & +112.0 & 0.0 & [17] \\
\hline PW91 & $\mathrm{BS} 2^{d}$ & Mn(porphyrin) & +80.0 & +51.0 & 0.0 & [33] \\
\hline LSD & $\mathrm{BS} 3^{e}$ & $\operatorname{Mn}(\text { salen })^{+}$ & -66.0 & 0.0 & +104.6 & [31] \\
\hline PW91 & BS3 & $\operatorname{Mn}(\text { salen })^{+}$ & -42.7 & 0.0 & +2.8 & [31] \\
\hline TPSS & BS3 & $\operatorname{Mn}(\text { salen })^{+}$ & -43.9 & 0.0 & +5.0 & [31] \\
\hline X3LYP & BS3 & $\operatorname{Mn}(\text { salen })^{+}$ & -18.7 & 0.0 & +82.3 & [31] \\
\hline X3LYP & $\mathrm{BS} 4{ }^{f}$ & $\operatorname{Mn}(\text { salen })^{+}$ & -19.5 & 0.0 & +80.5 & [31] \\
\hline X3LYP & BS5 $g$ & $\operatorname{Mn}(\text { salen })^{+}$ & -21.7 & 0.0 & +84.1 & [31] \\
\hline LSD & BS3 & $\mathrm{Mn}$ (salen) $\mathrm{Cl}$ & +6.1 & 0.0 & +240.5 & [31] \\
\hline PW91 & BS3 & $\mathrm{Mn}$ (salen)Cl & +17.9 & 0.0 & +225.7 & [31] \\
\hline TPSS & BS3 & $\mathrm{Mn}$ (salen)Cl & +22.7 & 0.0 & +224.9 & [31] \\
\hline X3LYP & BS3 & $\mathrm{Mn}$ (salen)Cl & +16.5 & 0.0 & +238.6 & [31] \\
\hline X3LYP & BS4 & $\mathrm{Mn}$ (salen)Cl & +24.1 & 0.0 & +236.8 & [31] \\
\hline X3LYP & BS5 & $\mathrm{Mn}$ (salen) $\mathrm{Cl}$ & +20.0 & 0.0 & +237.3 & [31] \\
\hline
\end{tabular}

${ }^{a}$ Double- $\zeta$ Valence with Polarization; ${ }^{b}$ mixed basis set: Triple- $\zeta$ Valence (TZV)on Mn, Split-Valence with Polarization (SVP)on other elements; ${ }^{c}$, not available; ${ }^{d}$, mixed basis set: Los Alamos National Lab Double- $\zeta$ Pseudo-Potential (LANL2DZ)on Mn, Dunning double- $\zeta$ on other elements; ${ }^{e}$ mixed basis set: TZV(2pf) for Mn; TZV for H; TZV(P) for other elements; $f$ mixed basis set: TZV(p) for Mn; Split-Valence (SV) for H; SVP for other elements; ${ }^{g}$ mixed basis set: $\mathrm{TZV}(2 \mathrm{pf})$ for $\mathrm{Mn}$; $\mathrm{TZV}(\mathrm{P})$ for $\mathrm{H}$; $\mathrm{TZV}(2 \mathrm{~d})$ for other elements.

On the other hand, manganese(V) (usually found in oxo-Mn(salen) species) has a $3 d^{2}$ configuration that allows one to postulate either a triplet or a singlet ground state. Again, the electronic structure of the $\mathrm{Mn}(\mathrm{V})$ atom will be influenced by the non-planarity of the salen ligand, the presence of the oxo- ligand and also the eventual presence of an axial ligand [11,23,31]. Because oxo-Mn(salen) complexes are extremely reactive, the absence of reliable experimental data has attracted the attention of many theoretical researchers. Some of their findings are summarized in Table 3, which depicts the relative gas-phase energies of the lowest-lying singlet triplet and quintuplet states of oxo-Mn complexes.

As can be seen from Tables 2 and 3, DFT has been the preferred theoretical approach for the study of the electronic structure and reactivity of $\mathrm{Mn}$ and Fe catalysts, as it offers reasonable approximations to the energy involved in electron correlation at a reasonable computational cost. 
Table 3. Relative gas-phase energies (in $\mathrm{kJ} \cdot \mathrm{mol}^{-1}$ ) of the singlet, triplet and quintet states of oxo-Mn complexes. The ground state in each entry is highlighted in bold.

\begin{tabular}{|c|c|c|c|c|c|c|c|}
\hline Method & Functional & Basis Set & Species & Singlet & Triplet & Quintet & Reference \\
\hline DFT & B3LYP & $\operatorname{DZVP}^{a}$ & ${\text { Oxo-Mn(acacen })^{+}}^{+}$ & 0.0 & -6.7 & +20.9 & [23] \\
\hline DFT & B3LYP & DZVP & Oxo-Mn(acacen)Cl & 0.0 & -33.9 & -39.3 & [23] \\
\hline $\operatorname{CCSD}(\mathrm{T})^{b}$ & - & DZVP & 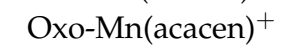 & 0.0 & +5.9 & +72.3 & [23] \\
\hline $\operatorname{CCSD}(\mathrm{T})$ & - & DZVP & Oxo-Mn(acacen)Cl & 0.0 & +60.6 & +45.6 & [23] \\
\hline DFT & BP86 & DZVP & Oxo-Mn(acacen)Cl & -59.8 & -32.6 & 0.0 & [32] \\
\hline $\mathrm{DMRG}^{-\mathrm{SCF}^{c}}$ & - & $6-31 G(d)$ & Oxo-Mn(acacen)Cl & 0.0 & -20.0 & +60.0 & [34] \\
\hline DFT & BP86 & $\mathrm{BS} 1^{d}$ & 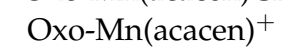 & 0.0 & +27.0 & +110.0 & [17] \\
\hline DFT & B3LYP & BS1 & Oxo-Mn(acacen $)^{+}$ & 0.0 & +11.0 & +40.0 & [17] \\
\hline DFT & PW91 & $\mathrm{BS} 2^{e}$ & Oxo-Mn(porphyrin) & 0.0 & +1.0 & +29.0 & [33] \\
\hline CASSCF $^{f}$ & - & $6-31 G(d)$ & Oxo-Mn(acacen $)^{+}$ & 0.0 & -12.1 & +165.9 & [35] \\
\hline MRMP2 $g$ & - & $6-31 G(d)$ & Oxo-Mn(acacen $)^{+}$ & 0.0 & -9.6 & +188.1 & [35] \\
\hline DFT & LSD & BS3 ${ }^{h}$ & ${\mathrm{Oxo}-\mathrm{Mn}(\text { salen})^{+}}^{+}$ & -16.0 & 0.0 & +99.2 & [31] \\
\hline DFT & PW91 & BS3 & Oxo-Mn(salen) ${ }^{+}$ & +2.8 & 0.0 & +69.6 & [31] \\
\hline DFT & TPSS & BS3 & ${\mathrm{Oxo}-\mathrm{Mn}(\text { salen})^{+}}^{+}$ & +5.0 & 0.0 & +62.7 & [31] \\
\hline DFT & X3LYP & BS3 & $\mathrm{Oxo}^{\mathrm{Mn}(\text { salen })^{+}}$ & +23.5 & 0.0 & +45.0 & [31] \\
\hline DFT & X3LYP & $\mathrm{BS} 4^{i}$ & Oxo-Mn(salen) $^{+}$ & +21.8 & 0.0 & +40.4 & [31] \\
\hline DFT & X3LYP & BS5 $^{j}$ & Oxo-Mn(salen) $^{+}$ & +24.9 & 0.0 & +46.5 & [31] \\
\hline DFT & LSD & BS3 & Oxo-Mn(salen)Cl & -11.9 & 0.0 & +232.4 & [31] \\
\hline DFT & PW91 & BS3 & Oxo-Mn(salen)Cl & +5.8 & 0.0 & +218.4 & [31] \\
\hline DFT & TPSS & BS3 & Oxo-Mn(salen)Cl & +7.4 & 0.0 & +219.9 & [31] \\
\hline DFT & X3LYP & BS3 & Oxo-Mn(salen)Cl & +7.8 & 0.0 & +232.3 & [31] \\
\hline DFT & X3LYP & BS4 & Oxo-Mn(salen)Cl & +13.5 & 0.0 & +229.3 & [31] \\
\hline DFT & X3LYP & BS5 & Oxo-Mn(salen)Cl & +7.7 & 0.0 & +231.4 & [31] \\
\hline
\end{tabular}

${ }^{a}$, Double- $\zeta$ Valence with Polarization; ${ }^{b}$, Coupled Cluster with Single and Double excitations and extrapolated Triple excitations; ${ }^{c}$, Density Matrix Renormalization Self-Consistent Field; ${ }^{d}$, mixed basis set: TZV on Mn, SVP on other elements; ${ }^{e}$, mixed basis set: LANL2DZ on Mn, Dunning double- $\zeta$ on other elements; $f$, Complete Active-Space Self-Consistent Field; ${ }^{g}$, Multi-Reference second-order Møller-Plesset; ${ }^{h}$, mixed basis set: TZV(2pf) for Mn; TZV for H; TZV(P) for other elements; ${ }^{i}$, mixed basis set: TZV(p) for Mn; SV for H; SVP for other elements; ${ }^{j}$, mixed basis set: $\mathrm{TZV}(2 \mathrm{pf})$ for $\mathrm{Mn}$; TZV $(\mathrm{P})$ for $\mathrm{H}$; TZV $(2 \mathrm{~d})$ for other elements.

Targeting Mn(salen)-catalyzed epoxidations, Abashkin et al. [23] confronted the results obtained from DFT with those obtained from Coupled Cluster (CC) theory, at the CCSD(T) (T, Tripe) level of approximation. The DFT calculations were performed using two different functionals, with a pure density functional (BP86) predicting a singlet ground state and a hybrid-GGA functional (B3LYP) predicting a quintuplet ground state. The results from CC calculations suggested a singlet ground state being preferred over states of higher spin multiplicity. However, the high cost of CC calculations forced Abashkin and co-workers to use Mn(acacen) (5) as a model for Mn(salen) (2). In this particular case, the use of truncated models may be regarded as a question of lesser importance, as both theoretical methods were applied to the same model compound. On the other hand, the chosen basis set and criterion used to limit the active space in CC calculations may have influenced their results. While DFT calculations were performed using a Split Valence (SV) basis set with some polarization, CC calculations were performed either using the same SV basis set or by applying a triple- $\zeta$ basis set for some atoms and restricting the active space of the CC procedure to only the most energetic occupied orbitals. Both procedures translate into additional truncations to the systems. Furthermore, neither the accuracy of DFT is fully explored using such a limited basis set, nor the CC method can assure reliable results with so many restrictions [36]. Moreover, the energy of the different states changes significantly when changing basis sets, indicating that the $\mathrm{CC}$ results are not converged with respect to the basis set [37]. More recently, CASSCF calculations on Mn(acacen) models were performed independently by Ivanic, Sears and their respective teams, arriving at different conclusions [35,38]. According to Ivanic et al. [35], oxo-Mn(salen) complexes have a triplet ground state, with the first excited singlet state lying about $10 \mathrm{~kJ} \cdot \mathrm{mol}^{-1}$ above it. On the other hand, the calculations performed by Sears and 
Sherril in 2006 [38] arrived at a singlet ground state with two nearly degenerated triplet states lying approximately $12 \mathrm{~kJ} \cdot \mathrm{mol}^{-1}$ above the ground state. In the same work, Sears and Sherril compared the results obtained using several density functionals to the results of their CASSCF calculations, and although they pointed out that non-hybrid functionals gave results in qualitative agreement with CASSCF, they were unable to conclude on the overall quality of DFT calculations. Both works rely on SV basis sets with minimal polarization; Ivanic further resorted to Effective Core Potentials (ECP) in order to allow more polarization to be used in the description of the manganese and chlorine atoms. This latter strategy was later used by Takatani, Sears and Sherril $[39,40]$ in the benchmark of several density functionals against state averaged CASSCF calculations using a cationic version of 5 (without the axial ligand). According to their results, DFT (particularly using hybrid density functionals) should provide reliable results $[39,40]$. However, their work also relies on SV basis sets with minimal polarization. What is more, the cationic model they use had already been considered unfit to give a meaningful representation of either $\mathrm{Mn}$ (acacen) $\mathrm{Cl}$ or proper $\mathrm{Mn}$ (salen) complexes [41,42]. As a result, the calculations by Sears and Sherril [39] (cationic model) invert the ordering of the spin states found years earlier by the same authors, on the neutral model 5 .

More recently, Ashley and Baik [43] conducted a CASPT2study on oxo-Mn species with the aim to better characterize the oxygen-manganese binding. The results from their post-HF calculations show that there is a modest di-radical character to the $\mathrm{Mn}^{\mathrm{V}}=\mathrm{O}$ bond. Surprisingly, this di-radical character is only partially related to the charge transfer between $\mathrm{Mn}$ and $\mathrm{O}$, showing that this diradical character, although being an indicator of transfer of charge between the two atoms, is not a necessary condition. Ashley and Baik [43] further accessed the behavior of DFT functionals regarding this aspect of manganese-oxygen binding, showing that hybrid functionals like B3LYP tend to overestimate the radical character of the $\mathrm{Mn}^{\mathrm{V}}=\mathrm{O}$ bond, whereas local functionals without exact $\mathrm{HF}$ exchange tend to underestimate it.

Regarding the use of truncated models and smaller basis sets, Teixeira et al. [44] recently addressed this problem by testing a number of different models for the Jacobsen catalyst (6), ranging from $\mathrm{Mn}$ (acacen) to the full catalyst, in both native and oxo-derived species, as well as using a number of different axial ligands. Their survey took into account geometrical parameters, as well as the charge and distribution of all species under study and used a Principal Component Analysis (PCA) to obtain some insight on the relative importance of each approximation on the overall description of these species. The results show that the choice of basis set has a huge impact on the resulting geometries and charge distribution, with Aldrich's def2-SVP (an SV basis set with polarization) behaving very differently from triple- $\zeta$ basis sets. Indeed, the impact of the basis set used in their DFT calculations surpassed that of opting for $\mathrm{Mn}$ (acacen) instead of a proper Mn(salen) complex as a model for the full Jacobsen catalyst. The use of truncated models and smaller basis sets is also reflected in the results collected in Tables 2 and 3, where the ordering of the singlet, triplet and quintuplet states in Mn(acacen) and oxo-Mn(acacen) follows a different pattern to that of $\mathrm{Mn}$ (salen) and its oxo-derivative.

\section{Insights on the Reaction Mechanism}

As stated by McGarrigle and Gilheany [11], one of the major advantages of theoretical studies is the ability to gain further insight into the reaction mechanism. This is most effective when more than one mechanism is proposed and surveyed, allowing researchers to choose the one that better explains the experimental evidence.

Experimental evidence gathered from studying $\mathrm{Cr}$ (salen) catalyzed epoxidations, and also the reactivity of cytochrome P450 [11] suggests that the catalytic cycle for most Mn and Fe catalysts is an oxygen-rebound one. As exemplified in Figure 3 for $\mathrm{Mn}(\mathrm{salen})$-type catalysts, this type of mechanism develops in two steps: the first being the oxidation of the catalyst using a terminal oxidant (such as $\mathrm{H}_{2} \mathrm{O}_{2}$, TBHP or $\mathrm{OCl}^{-}$) and the second being the oxygen-transfer step to the olefinic substrate [45]. It is generally believed that oxo-manganese or oxo-iron species are responsible for the epoxidation of the substrate, as similar species have been observed and characterized in chromium-salen-catalyzed 
epoxidations $[11,46]$. They are also proposed as intermediaries resulting from the dismutation of $\mathrm{H}_{2} \mathrm{O}_{2}$ in cytochrome P450 [47].

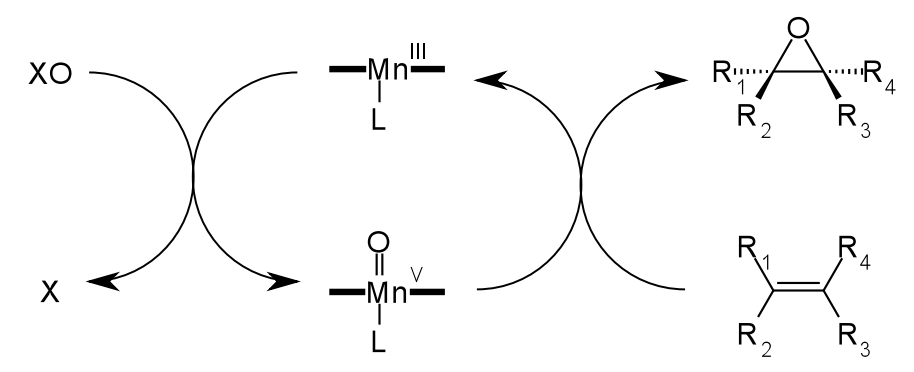

Figure 3. Oxygen-rebound mechanism for the Mn(salen)-catalyzed epoxidation of alkenes. The structure of the salen catalyst is simplified by a horizontal bar around the Mn atom; XO represents the terminal oxidant; and $\mathrm{L}$ is a generic axial ligand.

Abashkin and Burt conducted a DFT survey on the catalase-mimicking activity of $\mathrm{Mn}(\mathrm{salen}) \mathrm{Cl}$ [32], showing a viable route for the formation of oxo-Mn(salen) $\mathrm{Cl}$ from $\mathrm{Mn}$ (salen)Cl and $\mathrm{H}_{2} \mathrm{O}_{2}$. This is a concerted process, with the breaking of the $\mathrm{O}-\mathrm{O}$ peroxide bond, the oxidation of $\mathrm{Mn}$ and release of a water molecule occurring in a single step on the triplet state PES. In their turn, Cavallo and Jacobsen showed that oxo-Mn(acacen) $\mathrm{Cl}$ may be formed by a simple oxygen-transfer step between hypochlorite and $\mathrm{Mn}$ (acacen) $\mathrm{Cl}$ in aqueous solution [48]. More recently, Adhikary and co-workers [49] proposed the formation of an oxo-Fe(salen) species from iodosylbenzene (PhIO) and $\mathrm{Fe}(\mathrm{salen})$, which would be the active species in the iron-catalyzed epoxidation of stilbene. Similar intermediate species were also proposed by other authors $[13,33,50-54]$, indicating a strong consensus around the nature of the active catalyst of the epoxidation process.

Despite this, a limited number of sources has explored other routes, such as the formation of an hydroperoxo-Fe(porphyrin) complexes in P45models [55-59], in non-porphyrinic Fe catalysts [60-63] and also on Mn catalysts [64-67]. Nevertheless, the activity of such hydroperoxo species towards epoxidation reactions has been questioned by Shaik and co-workers [68], at least in models of cytochrome P450.

Because of this, most studies concerning the oxygen-transfer step of these epoxidations assume an active metal-oxo intermediate. As shown in Figure 4, a number of possible reaction paths are possible [45]. These include the formation of a metallaoxethane intermediate (Figure 4a), a concerted mechanism (Figure $4 \mathrm{~b}$ ), the formation of a five-membered ring structure including a nitrogen or oxygen atom of a spectator ligand, such as salen, or porphyrin (Figure $4 \mathrm{c}, \mathrm{d}$, respectively), or the formation of a carbon radical intermediate (Figure 4e) [34]. Each of these mechanisms allows the explanation of at least some of the experimental data available for these reactions, in particular the high enantioselectivity of Mn(salen)- and Fe(porphyrin)-catalyzed epoxidation of cis-olefins [23,50,69,70].

The stereoselectivity of these epoxidations provides further insights into the oxygen transfer mechanism. As can be inferred from Figure 4, Reaction Paths (a), (b), (c) and (d) should, in principle, lead exclusively to the epoxide that reflects the E/Zarrangement of the parent alkene (syn addition of the oxygen atom), whereas the product of an antiaddition is better accounted for by postulating the existence of a radical intermediate. The nature of the alkene substrate appears to have a strong influence on the outcome of $\mathrm{Mn}$ (salen)-catalyzed epoxidations: alkyl-substituted cis-alkenes usually attain high yields and stereoselectivities, leading to the hypothesis that such epoxidations may take place via a concerted mechanism (Figure $4 b$ ) $[9,10]$. On the other hand, the epoxidation of conjugated Z-alkenes produces mixtures of cis- and trans-epoxides, with the cis/trans ratio of the resulting epoxides depending on the nature of the substrate. Proponents of the metallaoxethane intermediate had argued that this decrease in stereoselectivity could emerge from the energy difference between two competing intermediates of that kind [71]. On the other hand, Jacobsen and co-workers proposed a carbon radical 
intermediate as a plausible intermediate in the epoxidation process (Figure 4e), yielding a simpler reaction model for this class of reactions [11].

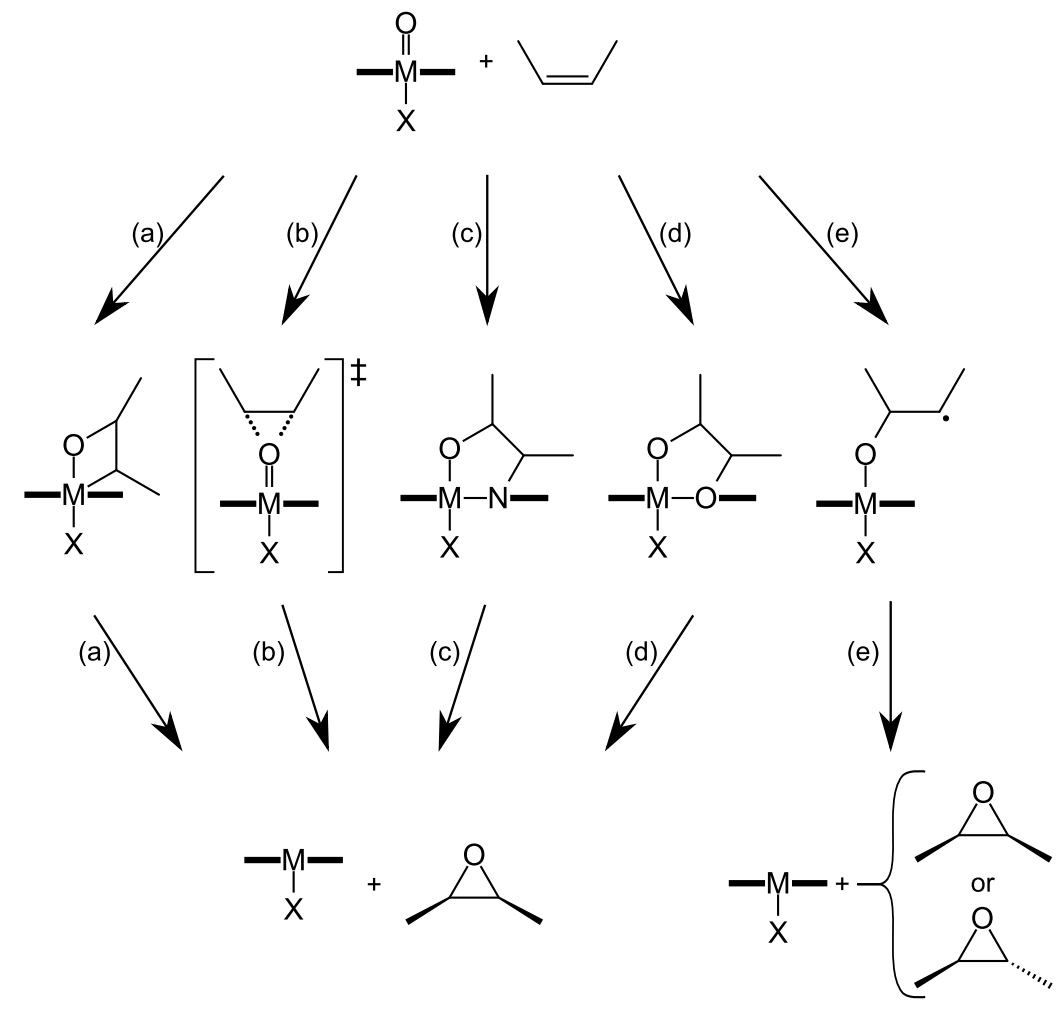

Figure 4. Generic representations of the most commonly-studied reaction paths for the oxygen atom transfer between oxo-manganese and oxo-iron complexes and a cis-alkene: metallaoxethane intermediate (a); concerted transition state (b); five-member ring intermediate using a nitrogen (c) or oxygen (d) from the main ligand; or via a carbon radical intermediate (e).

Despite explaining the lack of stereospecificity in the epoxidation of conjugated Z-alkenes, the carbon radical pathway has met some criticism. In the late 1990s, Linde et al. [72] used Mn(salen) complexes as catalysts in the epoxidation of phenyl-substituted vinylcyclopropanes, which serve as "radical clocks". These experiments allowed the rejection of a possible carbon radical, because no epimerization or cleavage of the cyclopropane was observed. However, further kinetic studies made by the same authors allowed them to reconsider the possible formation of radical intermediates. According to Linde et al. [72], the product of a syn addition is justified by a concerted, although possibly asynchronous, reaction mechanism. On the other hand, a radical intermediate may be the cause for the incomplete stereoselectivity of these catalysts.

Kürti et al. [73] rationalized the experimental data available at the time in order to propose an alternative model for this type of epoxidation reaction, mostly based on steric considerations. According to Kürti, electrostatic, van der Waals and $\pi-\pi$ interactions govern the stereoselectivity of $\mathrm{Mn}$ (salen) catalysts. Furthermore, the oxygen-transfer step from the active oxo-Mn(salen) intermediate to the alkene could occur either in a concerted manner or via a carbocation intermediate, similar to the radical intermediate depicted in Figure 4e. These two possibilities would be extreme idealized cases, and the stereospecificity for a particular substrate would be dictated by how close to either of these two extremes the actual reaction would occur.

One of the earliest theoretical studies on $\mathrm{Mn}$ (salen)-catalyzed epoxidations was conducted by Linde et al. in 1999 [74]. This work was performed using a cationic variant of the Mn(acacen) model (cf. Figure 2). Their findings allowed them to associate the reaction in the quintuplet surface to that of 
a concerted mechanism (Path (b) in Figure 4). In the triplet surface, however, the reaction mechanism would follow a radical intermediate (Path (e) in Figure 4). The authors then concluded that the reaction would begin by alkene attack on the triplet surface, followed by spin crossing to the quintuplet state. The stereospecificity of the reaction would be dictated by the amount of time the system was allowed to stay in the triplet state, due to the radical character of the first transition state in the triplet surface and the fact that formation of the epoxide is concerted and almost spontaneous in the quintuplet surface.

The work by Linde and co-workers was later criticized by Cavallo and Jacobsen and also by Abashkin and co-workers. Cavallo and Jacobsen [75] were able to devise a mechanism in which the oxygen transfer step occurs in the triplet surface, without the need for a spin-crossing path. The imperfect stereoselectivity is thus justified by the formation of a carbon radical species. In this carbon radical, the groups bonded to one of the carbons participating in the olefinic bond would be free to rotate, and the more stable the carbon radical would be, the less stereospecificity would be observed.

In their turn, Abashkin et al. [23] argued that the controversial spin crossing mechanism, as well as the ordering of the spin states obtained by Linde and co-workers were the result of using the cationic Mn(acacen) model, instead of the electronically neutral $\mathrm{Mn}$ (acacen)Cl. In an early stage, Abashkin and co-workers [23] favored the competing reaction channels scheme (originally proposed by Linde and co-workers, in their kinetic studies [72]) as an explanatory model for the stereoselectivity of $\mathrm{Mn}$ (salen)-catalyzed epoxidations. Their position was later revised after performing DFT calculations on a full Mn(salen) complex (cf. Formula 2 in Figure 1). In this latter work, the authors revisit the spin crossing mechanism, stating that the oxygen transfer step would start at the singlet ground state of the oxo-Mn(salen) complex leading to a concerted mechanism (Path (b) in Figure 4). However, because the activation energy in the singlet surface is too high, a spin crossing to the triplet surface was plausible. In the triplet surface, the oxygen transfer step occurs via a radical intermediate. Thus, the stereoselectivity of the reaction would depend on the likelihood of this spin crossing process, which in turn would be affected by the nature of the substrate and changes in the catalyst [69].

Cavallo and Jacobsen [17,41,42,48,75-77] have consistently championed the idea of a radical intermediate in the oxygen-transfer step of Mn(salen) epoxidations (Path (e) in Figure 4). Cavallo and Jacobsen explored three basic mechanisms depicted as Paths (a), (b) and (e) in Figure 4 using different density functionals and concluded that pure functionals, especially BP86, were best suited for the development of reaction models that conformed with the experimental observations. On the other hand, the popular hybrid density functional B3LYP tends to stabilize the states with higher multiplicity. This deviation was partially corrected, obtaining a better qualitative agreement with the pure density functionals, by using the modified functional B3LYP* [78] that uses a smaller weight of the Hartree-Fock exchange.

A radical intermediate has also been prescribed by Rich et al. [79] as the likely intermediate in the epoxidation of styrene catalyzed by a number of manganese(IV) complexes with spiroamine ligands, according to their DFT calculations (BP86) with implicit solvent corrections under the Polarizable Continuum Medium (PCM) framework.

More recently, Manrique et al. [80] also proposed the existence of a radical intermediate to justify the incomplete stereoselectivity for the epoxidation of $c i s-\beta$-methyl styrene by pyrazole-manganese(II) and pyrazole-manganese(III) catalysts. One peculiarity in this study is the fact that it uses a mixed level of theory: the relatively expensive geometry optimizations (including the search for transition state structures) and evaluation of the nuclear Hessian were done using a local density functional (M06L) in conjunction with a relatively small basis set (Aldrich's split-valence, with a more sophisticated relativistic SDDbasis set on Mn). These calculations provided the geometry and also the enthalpic and entropic corrections needed for calculating absolute Gibbs energies. However, the electronic component of the Gibbs energy was refined using single-point calculations performed with a functional that includes exact exchange (M06), a more flexible basis set (TZVP) and also the inclusion of solvent effects using PCM [80]. 
Using a combined QM/MM technique, Cavallo and Jacobsen not only stated the adequacy of $\mathrm{Mn}$ (acacen) complexes as surrogates of $\mathrm{Mn}$ (salen), but were also able to probe the different paths of approximation between oxo-Mn(salen) complexes and their substrate. They concluded on the importance of the bulky substituents on the diimine bridge and the 3,3' positions of the salen ligand, while stating the very limited steric effect of large substituents at the $5,5^{\prime}$ positions, in accordance with the experimental observations by Jacobsen, Katsuky and their respective co-workers [81]. According to the model proposed by Cavallo and Jacobsen $[17,75,76,81]$, the stereospecificity observed in this reaction derives from the relative stability of the radical intermediate: an unstable radical intermediate would promptly collapse in order to form the epoxide, whereas more stable intermediates would allow rotation of the substituents in one of the olefinic carbon atoms before the formation of the second $\mathrm{C}-\mathrm{O}$ bond. Spin crossing situations, as well as the possible existence of a metallaoxethane intermediate are discarded based on energetic grounds $[17,76,77,81]$. The crowning achievement of Cavallo and Jacobsen was the proposal of a model for the catalyzed ethylene epoxidation, arriving at the complete catalytic cycle depicted in Figure 5. This model for the Mn(salen) catalyzed epoxidation of ethene develops exclusively on the triplet surface [48]. However, the approach taken by Cavallo and Jacobsen to model $\mathrm{Mn}$ (salen) catalyzed epoxidations did not prove satisfactory for explaining the effect of axial coordination to the manganese atom over the development of the reaction course [77].

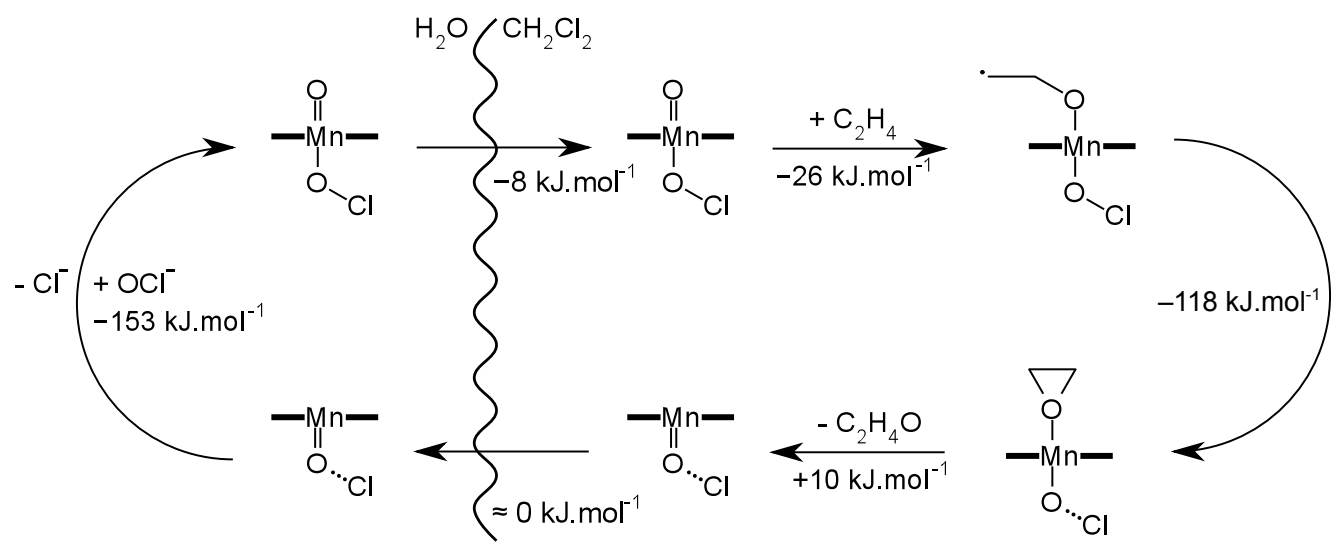

Figure 5. Catalytic cycle of $\mathrm{Mn}$ (acacen) $\mathrm{ClO}$ in a biphasic water/dichloromethane medium, adapted from Cavallo and Jacobsen [48]. The structure of the acacen moiety is represented in a simplified fashion, using black bars around the manganese atom.

Both the teams of Abashkin and Cavallo followed the ideas of Kochi and Jacobsen, using a planar model for Mn(salen). On the other hand Khavrutskii et al. [82] pursued the hypothesis of a non-planar, highly bent, conformation of the salen moiety in oxo-Mn(salen) complexes previously proposed by Katsuki in the 1990s [9]. Khavrutskii et al. [82] studied the epoxidation of ethylene using a proper Mn(salen) model (cf. Model 2, in Figure 1) and a peroxy acid $\left(\mathrm{CH}_{3} \mathrm{COOOH}\right)$ as the terminal oxidant. Their work is singular, as it considers the oxygen transfer step from either cis-oxo-Mn(salen) or trans-oxo-Mn(salen), depicted in Figure 6. Moreover, in this work, the energies of the molecular geometries at each stationary point (product, reactant or transition state) were refined using a basis set of triple- $\zeta$ and the B3LYP functional. Their results suggest that, starting from cis-oxo-Mn(salen), the oxygen transfer step may occur solely on the triplet surface, via a radical intermediate. Indeed, a similar radical intermediate was identified also in the quintuplet surface, but at higher energy. The same is also the general case for the reaction path starting from trans-oxo-Mn(salen). Despite this, in the particular case of imidazole-coordinated complexes, a concerted mechanism was observed in the quintuplet surface. In addition to this, the authors confirmed the cis form of oxo-Mn(salen) complexes as being more stable than their transisomers [82]. 
a)

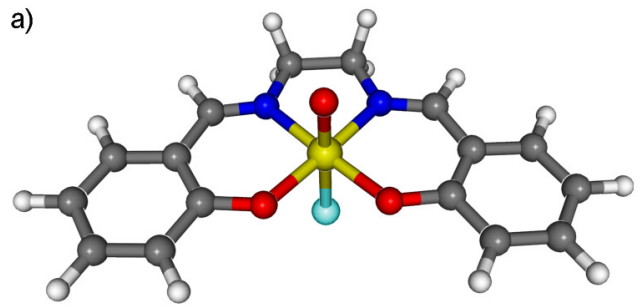

b)

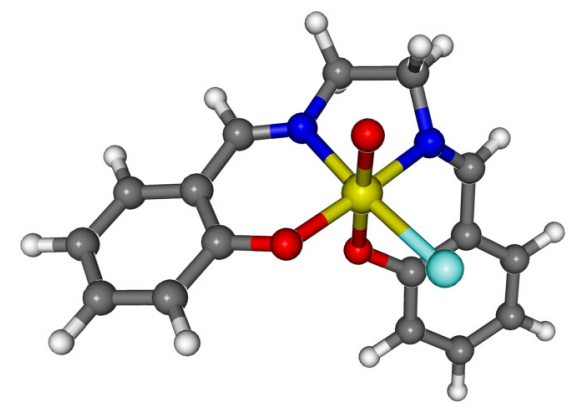

Figure 6. Cis (a) and trans (b) isomers of oxo-Mn(salen)Cl. In these representations, the carbon atoms are represented in grey, hydrogen in light grey, oxygen in red, nitrogen in blue, chloride in light blue and the manganese atoms as yellow spheres.

The behavior of immobilized Mn(salen) complexes was probed by Malek et al. [83,84] in two different studies. In the first study, Molecular Dynamics (MD) simulations were performed in which the movement of all atoms was treated using classical mechanics. Because bond-forming and bond-breaking situations cannot be accounted for in classical mechanics, the study focused on the minima found in the triplet surface by Cavallo and Jacobsen [48,76]. As MD is much less demanding than DFT and other first principles calculations (in terms of computational effort), the authors were able to explore the conformational space of these minima for the epoxidation of $Z$ - $\beta$-methylstyrene and $E-\beta$-methylstyrene catalyzed by the Jacobsen catalyst. Moreover, the catalyst was immobilized in a nanopore of silica MCM-41 using a phenoxyl group as a linker. Both outcomes of the epoxidation reaction (preservation or inversion of the arrangement of the methyl and phenyl groups of the alkene) were considered, and the chirality content at each of the different minima was evaluated using the continuous chirality measurements devised by Avnir [85]. Their main finding was that immobilization improved the chiral recognition of the catalyst and that the use of an E-alkene imparts greater asymmetric induction onto the immobilized catalyst [83].

A second study by Malek et al. [84] focused on the mechanism of the oxygen-transfer reaction using hybrid QM/MM calculations, in which part of the system was treated using DFT with an SV basis (except $\mathrm{Mn}$, which was treated using a triple- $\zeta$ basis). In order to accommodate the linker and the substrate in the DFT part of the system, an extreme truncation of the salen ligand was made, with only the nitrogen, oxygen and the two carbon atoms in the diimine bridge being treated under DFT. The remainder of the system (i.e., most of the Jacobsen catalyst, the linker and the silica MCM-41 pore) were treated classically, using Molecular Mechanics (MM). Despite the extreme approximations made, their results clearly suggest that the silica pore has an important effect in the orientation of the substrate towards the catalysts. What is more, the linker ligand bears a strong effect on the geometry of the active oxo-Mn(salen) complex, forcing the metal center to lay in the plane of the salen moiety and lengthening the bond between manganese and its oxo- ligand.

More recently, Oxford et al. [86,87] studied the conformation and reactivity of Mn(salen) complexes in Metal-Organic Frameworks (MOFs) using hybrid QM/MM. These calculations were done in a similar manner to those of Malek et al. [83,84], using the BP86 functional and a polarizable SV basis set on the QM part of the system (similarly to Malek et al., the Mn atom was treated using a triple- $\zeta$ basis). Initially, Oxford et al. investigated two partition schemes for defining the QM region: in one of those 
schemes, only the atoms belonging to the Mn(acacen) model (cf. Figure 2) were treated in the QM region, while in the other scheme, all of the salen moiety was included in the QM region. In both cases, the MOF was treated using classical MM. Regarding the electronic and steric effect of the substituents at the 5,5' positions of the salen ligand, the authors concluded that a correct description for the effect of these substituent required placing the conjugated $\pi$ system of the salen moiety in the QM region. More importantly, they achieved an interesting correlation between the Muliken charge on Mn and $\Delta E$ for the oxidation process [86], which would allow for a rapid virtual screening of several ligands with different substitution patterns at their $5,5^{\prime}$ positions. Oxford et al. [87] further went on studying the epoxidation of 2,2-dimethyl-2H-chromene using $\mathrm{Mn}$ (salen) immobilized in an MOF framework using the same QM/MM approach, but only treating the atoms in the Mn(acacen) model (as well as those from the substrate moiety) using QM. The calculated effect of immobilization of $\mathrm{Mn}$ (salen) in MOFs is much more pronounced than that determined by Malek et al. for MCM- 41 immobilized catalysts [84,87]. Oxford et al. further determined that, while the chirality content of the catalyst plays a major role determining its stereoselectivity, these two variables are not strongly correlated in the case of the MOF-immobilized catalysts, showing that the supporting media play an important role determining the selectivity of the hybrid catalysts, either by imposing conformational hindrances to the $\mathrm{Mn}$ (salen) moiety or by limiting the availability of paths for the substrate to approach the active catalyst [87].

\section{Comparison with Experimental Data}

The greatest test for a theoretical model is to provide insights that explain and allow one to predict the results from experiments. Fortunately, corroboration between experimentalists and theoretical chemists has become common in later years, and a significant number of sources attempts to compare the results from theoretical calculations with data obtained empirically. Moreover, whenever possible, theoretical chemists compare their results to previously published experimental data.

Iron-porphyrin-mediated epoxidations has attracted a number of important theoretical studies, with different approaches being used to mimic the environment of these iron complexes within cytochrome P450. A good benchmark for the validity of the theoretical approximations is to compare the predicted equilibrium geometries with those found by X-ray crystallographic studies of $\mathrm{P} 450$ and similar systems. As reported by Quiñonero et al. [62], B3LYP/LANL2DZ and B3LYP/6-311+G(d) $\mathrm{Fe}-\mathrm{N}$ distances in their iron-porphyrin complex models are in good agreement with those observed in P450. What is more, both levels of theory correctly predicted a quintuplet ground state for the tested complexes, which is also in agreement with experimental evidence. More recently, some studies reported similarly good agreement between experimentally-derived and theoretically-predicted equilibrium geometries for a number of iron-porphyrin complexes with different ligand compositions using B3LYP and PBE0 calculations. Similar results have been also reported by Sainna et al. $[60,88,89]$.

Kaczmarzyk et al. [90] used the OLYPfunctional with a triple- $\zeta$ polarized basis set to elucidate the Mössbauer spectra of (TPP) FeCl (in which TPP represents tetra-phenyl-porphyrin) and its fluoridated derivatives, showing very good agreement between DFT predictions and experimental data.

Another important source of validation for theoretical works is their ability to propose reactivity models that are in accordance with the experimental evidence. In this regard, de Visser et al. [91] reported that B3LYP can accurately predict energy barriers for the hydroxylation of anthracene catalyzed by non-heme iron(IV)-oxo oxidant, with an error of approximately $12 \mathrm{~kJ} \cdot \mathrm{mol}^{-1}$.

Kamachi et al. [57] further analyzed the epoxidation of ethylene catalyzed by an iron-porphyrin complex as a surrogate model for cytochrome P450. Their proposed model used an iron-hydroperoxide active species instead of the more common oxo- intermediates. The thermodynamic balances and energy barriers predicted by their calculations are in good agreement with the available experimental data, despite their use of B3LYP with a very modest basis set: compact effective potentials for Fe, a 1-2-1 triple- $\zeta$ basis set for S, 6-31G for nitrogen and oxygen atoms other atoms close to Fe and STO-3Gfor all other atoms [57]. 
Sometimes, theoretical findings are able to propose and explore novel reaction paths and intermediates not readily perceived in experimental studies. Such is the case of proposing an oxo-iron-porphyrin intermediate as the active species of cytochrome P450, which DFT and valence bond methods applied by Shaik and co-workers [68,92-94] demonstrated to be more active towards the epoxidation of olefins than the previously proposed iron-hydroperoxide intermediates. This further yielded novel models that better describe the product distribution of iron-porphyrin catalyzed epoxidations. Interestingly, Shaik and co-workers further proposed that the preference for $\mathrm{C}=\mathrm{C}$ epoxidation shown by $\mathrm{P} 450 \mathrm{CpdI}$ in theoretical studies results from not accounting for the polarizing environment found in P450. Thus, a more refined model, including polarization effects, was developed, which accounted for the available experimental observations $[95,96]$.

Regarding manganese-catalyzed epoxidations and their associated issues, Drzewiecka-Matuszek et al. [97] reported that Mn(III)porphyrin complexes have a high-spin ground state (quintuplet) using DFT calculations at the BP86/def-TZVP level of theory. Their results were in good agreement with the available experimental data. Eshtiagh-Hosseini et al. [51] used B3LYP $/ 6-311+G(d, p)$ to predict the IR, ${ }^{1} \mathrm{H}-\mathrm{NMR}$ and ${ }^{13} \mathrm{C}-\mathrm{NMR}$ spectra of 3,3'-dihydroxy-4,4'-[1,2-cyclohexanediyl-bis(nitrilomethylidyne)]-bis-phenol and its $\mathrm{Mn}$ (II) complex. Despite some deviations (within the expected error of this type of calculation [98]), the results from the DFT calculations were in good agreement with the experimental results, allowing the identification of the manganese complex and also the assignment of the peaks in the IR spectrum.

Rich et al. [79] also compared the structures predicted by DFT for their synthesized aminosprio-manganese(IV) complexes and against the results from $X$-ray spectroscopy. Despite the use of a pure functional (BP86) with a relatively small basis set of SV quality, the theoretically-predicted structures were in excellent agreement with their experimental measurements, with a root mean square deviation of $0.021 \AA$ on bond lengths and $0.7^{\circ}$ on bond angles.

In their study of permanganyl chloride-mediated epoxidations, Wistuba and Limberg [99] used DFT to help elucidate the nature of intermediate species found when monitoring the reaction using Infrared (IR) spectroscopy. The predicted frequencies were matched to unknown signals in the experimental IR, with remarkable accuracy. Their DFT calculation, at the B3LYP/6-311G(d) level of theory, further allowed the proposal of a complete mechanism for this type of Mn-catalyzed epoxidation.

Despite the limitations mentioned earlier in this work, the gathered knowledge shows that DFT methods are capable of providing remarkably good approximations for other properties of transition metal complexes, such as the prediction of spectroscopic properties. They also allow interesting insights into the catalytic cycle, yielding models that are in good accordance with the available experimental data. What is more, some of the most interesting accounts of good accordance between experimental data and theoretical predictions involve the use of rather limited basis sets, in an apparent contradiction to some of the previous studies [35,44]. However, a quantitative comparison between experimental data and theoretical predictions has eluded our bibliographical survey, apart from the comparisons encompassing spectroscopic data [51,90,99] and geometries [60,62,88,89].

\section{Other Epoxidation Catalysts: A Brief Overview}

The biological importance of cytochrome P450, as well as its role in the development of novel epoxidation catalysts justify the large number of theoretical and experimental studies devoted to iron-porphyrin systems. This predominance is shared with manganese-salen complexes, mainly due to the success of the Jacobsen-Katsuki epoxidation process. Still, the theoretical study of transition-metal catalyzed epoxidations goes far beyond the two major examples highlighted in this review $[100,101]$. A brief overview of the state of the art in the theoretical study of other transition metal-catalyzed epoxidations is thus provided here for completeness. The interested reader may find this section as a starting point to a more in-depth search of each of these systems. 
Chromium complexes, particularly chromium-salen, are known catalysts in epoxidation reactions, and their experimental study precedes that of their manganese counterparts [11]. Chromium-mediated epoxidations usually involve the formation oxo-chromium intermediates, which are stable enough to be isolated and studied. This facilitates comparing experimental data with theoretical predictions, as exemplified in Brandt et al. [102], where a coupled experimental and theoretical study of chromium-salen-mediated epoxidations allowed the successful characterization of the oxo-intermediate. Based on their DFT calculations, Brandt et al. [102] proposed that the oxygen transfer would occur via a radical intermediate, similarly to the reaction path described in Figure 4e. What is more, a spin-crossing situation was proposed, which would justify the relatively slow pace of chromium-catalyzed epoxidations. Similar results were later achieved by Venkataramanan et al. [103]. Despite this, the theoretical study of chromium-catalyzed epoxidations is substantially less explored than that of manganese or iron-catalyzed ones.

On the other hand, titanium, vanadium and molybdenum play an important role as catalysts in the Sharpless epoxidation process. Contrary to what is generally accepted for the Jacobsen-Katsuki epoxidation, the active epoxidizing agents in the Sharpless process are peroxo, hydro-peroxo or alkyl-peroxo-complexes [104]. Titanium and vanadium appear to bear similar chemical behavior, and both have attracted considerable attention in the last decade [105-110]. In general, the coordination sphere in both cases can be quite labile (especially in the case of vanadyl(IV) acetylacetonate, $\mathrm{VO}(\mathrm{acac})_{2}$ ) [104], which requires additional study of the effect of the different species available during the epoxidation process. In the case of vanadium, this was first systematically studied by Vandichel et al. [104], whereas the case of titanium was tackled earlier by Sever and Root [111]. Some theoretical studies on titanium-catalyzed epoxidations also focused on this metal's ability to efficiently catalyze the epoxidation of olefins when immobilized into a heterogeneous matrix, such as silicates [112] or polyoxometalates [113,114]. These theoretical studies follow the same trend observed in the case of manganese- and iron-catalyzed epoxidations, with a predominance of DFT, with B3LYP as the preferred density function. In this regard, Vandichel's work stands out due to the inclusion of Grimme's semi-empirical corrections for long-distance interactions [104].

Molybdenum, although similar to vanadium in many regards, bears some reactivity peculiarities that have been the focus of some theoretical studies. Perhaps the most distinctive feature of molybdenum is its ability to coordinate to the olefinic substrate $[107,115]$. While transition metals from the first row are often studied using a basis set that conveys information about all of their atomic orbitals, the large number of electrons and atomic orbitals present in the second and third row metals dictates the use of effective core potentials to model the kernel region. What is more, molybdenum is capable of forming complexes with multiple hydroperoxo and/or alkyl-peroxo ligands, increasing the number of available reactions paths to explore [116,117]. Recently, Morlot et al. [118] studied the activation of molybdenum catalysts by TBHP using both experimental and DFT methods. They concluded that the activation of TBHP is mostly due to a small interaction between Mo and one of the oxygen atoms of TBHP and also the formation of a hydrogen bond between TBHP's hydrogen atom (in the hydroperoxide moiety) and one of the ligands in the coordination sphere of Mo [118].

A large number of theoretical works has been devoted to ruthenium-catalyzed epoxidations. Being in the second row of the transition metal series, and being located bellow iron, ruthenium systems often share some similarities to their iron counterparts, having attained the attention of several researchers $[119,120]$. Unlike iron, di-nuclear ruthenium complexes are common and have been extensively studied using DFT methods [121,122].

Cobalt systems are not usually regarded as epoxidizing catalysts, but they do serve an important purpose as catalytic agents in epoxide ring-opening processes [3]. Cobalt-salen complexes with stereogenic centers are often used in the hydrolytic kinetic resolution of terminal epoxides, and their reactivity has been the subject of some theoretical studies [123].

Other interesting catalytic systems that have attained the attention of some theoretical studies include the catalytic behavior of immobilized $\mathrm{NiO}$ nanoparticles [124]; the epoxidation of propylene 
on the surface of $\mathrm{CuO}$, which has been studied by Düzenli et al. [125] using plane-wave DFT; the use of palladium(II) complexes in the epoxidation of olefins [126,127]; and also the epoxidation of ethylene using a bi-metallic palladium-silver catalyst [128].

Rare-earth catalyzed epoxidations (the Shibasaki process) have attained some attention in recent years [129], but to the best of our efforts no theoretical studies on this subject have been published at the time of writing. On the other hand, hafnium-catalyzed epoxidations using molecular oxygen as the terminal oxidant have been recently studied by Yang and Manz [130] using DFT calculations. Tungsten and niobium systems have also attracted some attention in recent years [131]; however, their theoretical study has been limited.

\section{Perspectives for Future Developments: SWOT Analysis}

In this review, we highlighted some of the most interesting advances provided by theoretical studies in Mn- and Fe-catalyzed epoxidations Particular emphasis was put on Mn(salen) and $\mathrm{Fe}$ (porphyrin) catalysts, as they are the most commonly-found systems, providing a sufficiently large number of studies to detect trends and their evolution over time. As some interesting trends were noticed, the most prominent of which are summarized in the form of a strengths, weaknesses, opportunities and threats framework (SWOT analysis in enterprise terminology). In order to minimize the subjective nature of a SWOT analysis, we define, within the context of this review, strengths as issues or problems for which there is a positive consensus on the quality of the theoretical results or for which there is a strong agreement between theoretical predictions and experimental evidence, allowing the use of theoretical methods to make predictive surveys for further enhancing the design of these catalysts. Weaknesses are defined as aspects in which a clear consensus on the quality of the theoretical results is lacking or in which unsolved fundamental issues have been identified. On the other hand, opportunities are identified as emergent technologies and/or areas of research that may provide new insights on the subject at hand. In their turn, threats are usually defined as external factors that may be barriers to the achievement of the goals. Apart from societal and/or political aspects (which are beyond the scope of this review), we identify threats as areas or matters that have the potential to stall or discredit novel theoretical studies. The evaluation of these four aspects is done in the hope of providing guidelines upon which new lines of research may emerge.

\subsection{Strengths}

Perhaps the most surprising outcome of this survey is the relatively high accordance between experimental data and the predictions provided by theoretical models. This is particularly noticeable in the case of spectroscopic data $[51,90,99]$ and equilibrium geometries $[60,62,79,88,89]$. What is more, the level of theory used in such models is sometimes bellow par (usually DFT calculations with B3LYP and a relatively small basis set). Nevertheless, the quality of the predictions is proof of the ability of theoretical models to accurately predict physical properties of the Mn and Fe complexes involved in epoxidation reactions. Thus, the use of theoretical models in the identification of intermediate species and products appears to be well validated.

The validation of theoretically-predicted reaction mechanisms is strongly hindered by the lack of quantifiable evidence. Despite this, the models published in the last 20 years gravitate around a common model for both $\mathrm{Mn}$ - and Fe-catalyzed epoxidations; namely, the use of oxo-Mn and oxo-Fe species as the active forms of the catalyst $[23,32,68,69,92-94,132]$, the avoidance of a spin-crossing mechanism $[42,48,76]$ and the possible role of a radical intermediate as a putative explanation for the incomplete stereoselectivity of these reactions $[76,82]$. It should be noted that many of these insights on the reaction mechanism are subtle enough to elude most of the standard kinetic studies. Despite these successes, the challenge to apply this knowledge to the ab initio design of novel catalysts remains unchecked. 


\subsection{Weaknesses}

Despite what was suggested by our initial statistical meta-analysis, most studies rely only on B3LYP calculations. This is partly compensated by some studies that benchmark their findings across a large number of density functionals $[17,31,34,133]$ (and eventually, other theoretical methods $[23,34,35,134])$. Moreover, most works accessing the performance of B3LYP acknowledge its limitations when applied to transition metal chemistry. On the other hand, the great majority of works comparing between experiment and theoretical predictions use B3LYP. One possible explanation may come from the work by Teixeira et al. [31], where, despite changes in the energy ordering of the different spin states of $\mathrm{Mn}$ (salen) complexes, the effective atomic charges provided by a natural population analysis of these complexes vary very little with respect to the level of theory used in the energy calculation. These results are further corroborated by a topological analysis of the electron density using Bader's Quantum Theory of Atoms In Molecules (QTAIM) [31]. Since the distribution of the electron density governs a great number of physical properties, it is likely that the limitations of B3LYP may be negligible in many cases.

A more serious limitation of the early theoretical studies is the use of truncation as a mean to keep DFT calculations within a reasonable computational cost, given the state of computer technology at the time. These truncations may be in the form of using smaller model transition metal complexes or by using small basis sets, which cannot fully exploit the accuracy of the theoretical framework applied. The consequences of such simplifications have been explored recently [44,135] and should grant the need to re-address the calculations made by previous works. In this regard, it is worth mentioning that a clear trend in newer works is replacing the use of smaller, less flexible basis sets with larger basis sets, usually triple- $\zeta$ quality ones, with polarization on all atoms.

Despite this, an interesting strategy to achieve a compromise between accuracy and the economy of computational effort is exemplified in the work of Manrique et al. [80], where the most computationally-demanding tasks (geometry optimization and evaluation of the vibrational spectra) are done at a lower level of theory, providing the thermal and zero-point energy components of the absolute enthalpies and Gibbs energies. In their turn, the electronic energy is calculated at a higher (more expensive) level of theory at a fixed geometry, with the eventual inclusion of solvent effects using the PCM framework. This strategy is well established, and can be traced back to Pople's Gnmethods [136]. It should give accurate thermodynamic balances and activation barriers, provided that the inclusion of solvent effects and/or the change in the level of theory does not affect significantly the local shape of the PES (which could carry important changes in the equilibrium geometry and energetic corrections).

\subsection{Opportunities}

Historically, one of the major limitations facing the theoretical study of Mn and Fe catalyzed epoxidations has been the size of the transition metal complexes involved in such reactions. This has prevented the use of highly accurate ab initio methods to study the structure of the intervening species. It has hindered the use of DFT as the engine for studying more interesting details of these reactions, such as the effect of the protein moiety in cytochrome P450 and also the effect of confinement in immobilized catalysts. Moreover, this has brought forward a number of truncation schemes, such as the use of $\mathrm{Mn}$ (acacen) as a model for the Jacobsen catalyst or the use of very small basis sets in DFT calculations.

The idea of re-purposing Graphic Processing Units (GPU) for general-purpose computations has been championed by Martínez and co-workers [137]. The initial success of Martínez's attempts brought forward a happy confluence of interests between industry and academia, with most high-end consumer-grade graphics cards being able to run general-purpose calculations using either NVidia's proprietary CUDA platform or the Open Computing Language (OpenCL) platform. Due to the floating point precision of the GPU architecture, their use was first restricted to classical molecular mechanics simulations. Nevertheless, in recent years, the availability of high-accuracy floating point operations 
in high-end consumer-grade GPUs (as well as newer, computational-specific GPUs) prompted the development of new codes, enabling the computation of DFT energies and nuclear gradients [138]. More recently, ab initio post-Hartree-Fock methods have become available on GPU platforms, making MP2calculations available to systems with over 150 atoms [139] and Full Configuration Interaction (FCI) calculations available to nano-sized silica particles (with up to $72 \mathrm{Si}$ atoms), albeit using a modest 6-31G(d,p) basis set [140]. These new technologies open a new array of opportunities for exploring $\mathrm{Mn}$ and Fe catalyzed epoxidations, which remains unexplored.

While GPU-accelerated calculations rely on the paradigm of increasing the power of the machinery to forward the study of larger systems, another approach already taken by a number of theoreticians has been the implementation of efficient linear-scaling algorithms. One such method is the so-called Resolution of Identity (RI) method in which charge distributions arising from products of basis functions are approximated by a linear combination of auxiliary basis functions. This strategy effectively saves computational effort when calculating the Coulombic repulsion term in pure DFT functionals [141]. Hybrid DFT methods can also use a variant of the RI algorithm to achieve near-linear scaling in the calculation of the exact Hartree-Fock exchange term, in which the exchange integral is computed from fitting an analytical expression for the electron density instead of the usual numerical integration scheme [142]. Such methods have the advantage of being easily implemented into existing quantum chemistry software codes. Moreover, no specific hardware is required to take advantage of these methods. Drzewiecka-Matuszek et al. [97] used the RI approximation in their study of the reaction between $\mathrm{Mn}$ (porphyrin) and $\mathrm{H}_{2} \mathrm{O}_{2}$, allowing the formulation of a reactivity model, which is in line with the available experimental data. More recently, Teixeira et al. $[31,44,135]$ used these linear-scaling strategies in their study of the charge and spin distributions in $\mathrm{Mn}(\mathrm{salen})$ and related complexes (including their oxo-derivatives). Here, the results obtained by these authors are in good accordance with those obtained previously without the use of such approximations $[17,31,37,82]$.

In summary, linear scaling methods have the potential to dramatically lower the computational cost of calculations and allow larger systems to be studied at a decent level of theory. Furthermore, the errors introduced by these strategies appear to be negligible. Thus, it is likely that linear-scaling methods (perhaps even in conjunction with the use of GPUs) will become standard in the near future.

\subsection{Threats}

As computer technology evolves, more complex machinery becomes available. As stated above, this presents the opportunity to perform calculations on larger systems. The increasing complexity of the machines required to perform computations in large systems (specially ab initio calculations) uncovers a new type of problem to which the theoretical chemistry community afforded to be oblivious to until recently. These come in the form of Silent Data Corruption (SDC) issues, which can potentially cause erroneous results or software failures. Such errors are often related to erroneous bit-flip operations in the computing environment and may occur in any hardware platform, although with an extremely low probability. This is unlikely, as an SDC-causing error may be for one processing unit; complex computers with multiple processing units (either CPU or GPU) multiply that probability to an extent where it might become a serious threat to the scientific value of the theoretical and computational work. Fortunately, this issue has been addressed recently in a recent work by van Dam et al. [143] where a first approach for the detection and correction of SDC errors was first explained and tested on a number of computational tasks common to most ab initio calculations, particularly HF calculations. Their findings show that in a significant number of cases, an SDC error was capable of causing the HF calculations to fail (aborting the calculation). When an SDC error was not sufficient to cause the calculation to abort, the iterative nature of the HF method allowed for the affected calculation to "heal" itself from the error. With the expected increase in computer complexity, it is vital that this type of study be expanded to other first-principles calculations. At the same time, it is important that such questions are taken into consideration in the planning, execution and analysis of large calculations. 


\section{Conclusions}

The outlook for the theoretical modeling of manganese and iron-catalyzed epoxidations appears to be promising. Despite some limitations of the early works, the knowledge already gathered grants some confidence on the inner workings of the catalytic cycle of the most common epoxidizing catalysts. At the same time, the development of new computer technology and software algorithms opens the possibility of studying these reactions using models that better mimic the chemical environments found in experimental conditions.

After analysis of the available data, it is likely that the theoretical study of these catalysts will be more integrated with the experimental study of olefin epoxidations, either as support calculations to elucidate spectroscopic and X-ray data or as a means to test different hypotheses concerning the catalytic cycle of a particular catalyst.

Despite this, a number of theoretical challenges remains, such as the benchmark of DFT calculations against post-HF methods in large catalysts, the assessment of the effect of truncation in the reaction mechanism of $\mathrm{Mn}$ (salen) catalysts and also the development of predictive tools that will allow the ab initio design and fine-tuning of novel catalysts.

Acknowledgments: This work had the financial support of Fundação para a Ciência e a Tecnologia (FCT/MEC) through national funds and co-financed by FEDER, under the Partnership Agreement PT2020 (Projects UID/QUI/50006/2013 and POCI/01/0145/FEDER/007265). MNDSCfurther acknowledges FCT/MEC for the Sabbatical Leave Grant SFRH/BSAB/127789/2016.

Author Contributions: M.N.D.S.C. defined the concept, objectives and scope of this work; as well as the critical revision of the draft. F.T. was responsible for the data collection, analysis and interpretation, as well as drafting the manuscript.

Conflicts of Interest: The authors declare no conflict of interest.

\section{References}

1. Chandrachud, P.P.; Jenkins, D.M. High valent Fe-IV chemistry in sustainable oxidation catalysis. Tetrahedron Lett. 2015, 56, 2369-2376.

2. Webb, M.A.; Jung, Y.; Pesko, D.M.; Savoie, B.M.; Yamamoto, U.; Coates, G.W.; Balsara, N.P.; Wang, Z.G.; Miller, T.F. Systematic Computational and Experimental Investigation of Lithium-Ion Transport Mechanisms in Polyester-Based Polymer Electrolytes. ACS Cent. Sci. 2015, 1, 198-205.

3. Ahmed, S.M.; Poater, A.; Childers, M.I.; Widger, P.C.B.; LaPointe, A.M.; Lobkovsky, E.B.; Coates, G.W.; Cavallo, L. Enantioselective Polymerization of Epoxides Using Biaryl-Linked Bimetallic Cobalt Catalysts: A Mechanistic Study. J. Am. Chem. Soc. 2013, 135, 18901-18911.

4. Freire, C.; Pereira, C.; Rebelo, S. Green Oxidation Catalysis with Metal Complexes: From Bulk to Nano Recyclable Hybrid Catalysts. In Catalysis: Volume 24; The Royal Society of Chemistry: London, UK, 2012; Volume 24, pp. 116-203.

5. Lane, B.S.; Burgess, K. Metal-Catalyzed Epoxidations of Alkenes with Hydrogen Peroxide. Chem. Rev. 2003, 103, 2457-2474.

6. Liu, Y.; Murata, K.; Inaba, M. Direct epoxidation of propylene by molecular oxygen over a catalyst system containing palladium and a peroxo-heteropoly compound in methanol. Chem. Commun. 2004, 2004, 582-583.

7. Modi, A.R.; Dawson, J.H. Oxidizing Intermediates in P450 Catalysis: A Case for Multiple Oxidants. In Monooxygenase, Peroxidase and Peroxygenase Properties and Mechanisms of Cytochrome P450; Hrycay, G.E., Bandiera, M.S., Eds.; Springer: Cham, Switzerland, 2015; pp. 63-81.

8. Meunier, B. Metalloporphyrins as versatile catalysts for oxidation reactions and oxidative DNA cleavage. Chem. Rev. 1992, 92, 1411-1456.

9. Katsuki, T. Mn-salen Catalyst, Competitor of Enzymes, for Asymmetric Epoxidation. J. Mol. Catal. A Chem. 1996, 113, 87-107.

10. Dalton, C.T.; Ryan, K.M.; Wall, V.M.; Bousquet, C.; Gilheany, D.G. Recent progress towards the understanding of metal-salen catalyzed asymmetric alkene epoxidation. Top. Catal. 1998, 5, 75-91.

11. McGarrigle, E.M.; Gilheany, D.G. Chromium and Manganese-salen Promoted Epoxidation of Alkenes. Chem. Rev. 2005, 105, 1563-1602. 
12. He, J.; Xu, Z.G.; Xu, X.; Gong, L.Z.; Mahmood, M.H.; Liu, H.Y. Reactivity of (oxo)manganese(V) corroles in one-electron redox state: Insights from conceptual DFT and transition state calculations. J. Porphyr. Phthalocyanines 2013, 17, 1196-1203.

13. Sankaralingam, M.; Palaniandavar, M. Tuning the olefin epoxidation by manganese(iii) complexes of bisphenolate ligands: Effect of Lewis basicity of ligands on reactivity. Dalton Trans. 2014, 43, 538-550.

14. Shaik, S.; Cohen, S.; Wang, Y.; Chen, H.; Kumar, D.; Thiel, W. P450 Enzymes: Their Structure, Reactivity, and Selectivity Modeled by QM/MM Calculations. Chem. Rev. 2010, 110, 949-1017.

15. Simões, M.M.Q.; Neves, C.M.B.; Pires, S.M.G.; Neves, M.G.P.M.S.; Cavaleiro, J.A.S. Mimicking P-450 processes and the use of metalloporphyrins. Pure Appl. Chem. 2013, 85, 1671-1681.

16. Groves, J.T. Reactivity and mechanisms of metalloporphyrin- catalyzed oxidations. J. Porphyr. Phthalocyanines 2000, 4, 350-352.

17. Cavallo, L.; Jacobsen, H. Transition Metal Mediated Epoxidation as Test Case for the Performance of Different Density Functionals: A Computational Study. J. Phys. Chem. A 2003, 107, 5466-5471.

18. Kavousi, H.; Rezaeifard, A.; Raissi, H.; Jafarpour, M. A DFT investigation of axial N -donor ligands effects on the high valent manganese-oxo meso -tetraphenyl porphyrin. J. Porphyr. Phthalocyanines 2015, 19, 651-662.

19. Hai-Yang, L.; Li, L.; Xiao, Y.; Xiang-Li, W.; Zhi-Guang, X.; Shi-Jun, L.; Chi-Kwong, C. DFT calculations on manganese(III)5,10,15-tris(pentafluorophenyl)-corrole. Acta Phys. Chim. Sin. 2008, 24, 1602-1608.

20. Li-Zhen, G.; Zhi-Guang, X.; Xuan, X.; Jing, H.; Qi, W.; Hai-Yang, L. Axial Coordination Behavior of Corrole $\mathrm{Mn}$ and $\mathrm{Mn}^{V} \mathrm{O}$ Complexes with N-Based Ligands. Acta Phys. Chim. Sin. 2014, 30, 265-272.

21. Xiao-Hui, Z.; Zhi-Guang, X.; Li-Zhen, G.; Xuan, X.; Gui-Xian, S.; Hua-Bin, C.; Hai-Yang, L. Stability of trans-Dioxo Manganese(V) Corrole Complex. Acta Phys. Chim. Sin. 2015, 31, 1069-1076.

22. R Core Team. R: A Language and Environment for Statistical Computing; R Foundation for Statistical Computing: Vienna, Austria, 2013.

23. Abashkin, Y.G.; Collins, J.R.; Burt, S.K. (Salen)Mn(III)-Catalyzed Epoxidation Reaction as a Multichannel Process with Different Spin States. Electronic Tuning of Asymmetric Catalysis: A Theoretical Study. Inorg. Chem. 2001, 40, 4040-4048.

24. Zhao, Y.; Truhlar, D.G. The M06 suite of density functionals for main group thermochemistry, thermochemical kinetics, noncovalent interactions, excited states, and transition elements: Two new functionals and systematic testing of four M06-class functionals and 12 other functionals. Theor. Chem. Acc. 2008, 120, 215-241.

25. Nemeth, L.; Bare, S.R. Science and Technology of Framework Metal-Containing Zeotype Catalysts. In ADVANCES IN CATALYSIS, VOL 57; Advances in Catalysis; Jentoft, F., Ed.; Elsevier BV: Amsterdam, The Netherlands, 2014; Volume 57, pp. 1-97.

26. Pereira, C.; Pereira, A.M.; Quaresma, P.; Tavares, P.B.; Pereira, E.; Araújo, J.P.; Freire, C. Superparamagnetic $\mathrm{Fe}_{2} \mathrm{O}_{3} @ \mathrm{SiO}_{2}$ nanoparticles: A novel support for the immobilization of [VO(acac $\left.)_{2}\right]$. Dalton Trans. 2010, 39, 2842-2854.

27. Silva, A.C.; Cepera, R.M.; Pereira, M.C.; Lima, D.Q.; Fabris, J.D.; Oliveira, L.C. Heterogeneous catalyst based on peroxo-niobium complexes immobilized over iron oxide for organic oxidation in water. Appl. Catal. B 2011, 107, 237-244.

28. Pospisil, P.J.; Carsten, D.H.; Jacobsen, E.N. X-ray Structural Studies of Highly Enantioselective Mn(salen) Epoxidation Catalysts. Chem. Eur. J. 1996, 2, 974-980.

29. Bryliakov, K.P.; Babushkin, D.E.; Talsi, E.P. Detection of $\{$ EPR $\}$ Spectra in $S=2$ States of MnIII(salen) Complexes. Mendeleev Commun. 1999, 9, 29-31.

30. Campbell, K.A.; Lashley, M.R.; Wyatt, J.K.; Nantz, M.H.; Britt, R.D. Dual-Mode EPR Study of Mn(III) Salen and the Mn(III) Salen-Catalyzed Epoxidation of cis- $\beta$-Methylstyrene. J. Am. Chem. Soc. 2001, 123, 5710-5719.

31. Teixeira, F.; Mosquera, R.A.; Melo, A.; Freire, C.; Cordeiro, M.N.D.S. Charge Distribution in Mn(salen) Complexes. Int. J. Quantum Chem. 2014, 114, 525-533.

32. Abashkin, Y.G.; Burt, S.K. (Salen)Mn(III) Compound as a Nonpeptidyl Mimic of Catalase: DFT Study of the Metal Oxidation by a Peroxide Molecule. J. Phys. Chem. B 2004, 108, 2708-2711.

33. Curet-Arana, M.C.; Emberger, G.A.; Broadbelt, L.J.; Snurr, R.Q. Quantum chemical determination of stable intermediates for alkene epoxidation with Mn-porphyrin catalysts. J. Mol. Catal. A Chem. 2008, 285, 120-127.

34. Bogaerts, T.; Wouters, S.; Voort, P.V.D.; Speybroeck, V.V. Mechanistic Investigation on Oxygen Transfer with the Manganese-Salen Complex. ChemCatChem 2015, 7, 2711-2719. 
35. Ivanic, J.; Collins, J.R.; Burt, S.K. Theoretical study of the low lying electronic states of oxoX(salen) $(\mathrm{X}=\mathrm{Mn}$, $\mathrm{Mn}^{-}, \mathrm{Fe}$, and $\mathrm{Cr}^{-}$) complexes. J. Phys. Chem. A 2004, 108, 2314-2323.

36. Neese, F. Prediction of Molecular Properties and Molecular Spectroscopy with Density Functional Theory: From Fundamental Theory to Exchange-coupling. Coord. Chem. Rev. 2009, 253, 526-563.

37. Khavrutskii, I.V.; Musaev, D.G.; Morokuma, K. Structure, Stability, and Electronic and NMR Properties of Various Oxo- and Nitrido-Derivatives of [L(Salen) $\mathrm{Mn}(\mathrm{III})]^{+}$, Where $\mathrm{L}=$ None and Imidazole. A Density Functional Study. Inorg. Chem. 2003, 42, 2606-2621.

38. Sears, J.S.; Sherrill, C.D. The Electronic Structure of oxo-Mn(salen): Single-reference and Multireference Approaches. J. Chem. Phys. 2006, 124, 144314, doi10.1063/1.2187974.

39. Takatani, T.; Sears, J.S.; Sherrill, C.D. Assessing the Performance of Density Functional Theory for the Electronic Structure of Metal-Salens: The d6-Metals. J. Phys. Chem. A 2009, 113, 9231-9236.

40. Takatani, T.; Sears, J.S.; Sherrill, C.D. Assessing the Performance of Density Functional Theory for the Electronic Structure of Metal-Salens: The M06 Suite of Functionals and the d4-Metals. J. Phys. Chem. A 2010, 114, 11714-11718.

41. Cavallo, L.; Jacobsen, H. Electronic Effects in (salen)Mn-Based Epoxidation Catalysts. J. Org. Chem. 2003, $68,6202-6207$.

42. Jacobsen, H.; Cavallo, L. Re-evaluation of the Mn(salen) Mediated Epoxidation of Alkenes by Means of the B3LYP* Density Functional. Phys. Chem. Chem. Phys. 2004, 6, 3747-3753.

43. Ashley, D.C.; Baik, M.H. The Electronic Structure of $[\mathrm{Mn}(\mathrm{V})=\mathrm{O}]$ : What is the Connection between Oxyl Radical Character, Physical Oxidation State, and Reactivity? ACS Catal. 2016, 6, 7202-7216.

44. Teixeira, F.; Mosquera, R.A.; Melo, A.; Freire, C.; Cordeiro, M.N.D.S. Principal Component Analysis of Mn(salen) Catalysts. Phys. Chem. Chem. Phys. 2014, 16, 25364-25376.

45. Linker, T. The Jacobsen-Katsuki Epoxidation and Its Controversial Mechanism. Angew. Chem. Int. Ed. Engl. 1997, 36, 2060-2062.

46. Bautz, J.; Comba, P.; Lopezrden, C.; Menzel, M.; Rajaraman, G. Biomimetic High-Valent Non-Heme Iron Oxidants for the cis-Dihydroxylation and Epoxidation of Olefins. Angew. Chem. Int. Ed. 2007, 46, 8067-8070.

47. Kumar, D.; Tahsini, L.; de Visser, S.P.; Kang, H.Y.; Kim, S.J.; Nam, W. Effect of Porphyrin Ligands on the Regioselective Dehydrogenation versus Epoxidation of Olefins by Oxoiron(IV) Mimics of Cytochrome P450. J. Phys. Chem. A 2009, 113, 11713-11722.

48. Cavallo, L.; Jacobsen, H. Toward a Catalytic Cycle for the Mn-Salen Mediated Alkene Epoxidation: A Computational Approach. Inorg. Chem. 2004, 43, 2175-2182.

49. Adhikary, J.; Datta, A.; Dasgupta, S.; Chakraborty, A.; Menéndez, M.I.; Chattopadhyay, T. Development of an efficient magnetically separable nanocatalyst: Theoretical approach on the role of the ligand backbone on epoxidation capability. RSC Adv. 2015, 5, 92634-92647.

50. Benet-Buchholz, J.; Comba, P.; Llobet, A.; Roeser, S.; Vadivelu, P.; Wadepohl, H.; Wiesner, S. Iron vs. ruthenium-A comparison of the stereoselectivity in catalytic olefin epoxidation. Dalton Trans. 2009, 5910-5923.

51. Eshtiagh-Hosseini, H.; Beyramabadi, S.A.; Mirzaei, M.; Morsali, A.; Salimi, A.R.; Naseri, M.A. 3,3'-dihydroxy-4,4'-[1,2-cyclohexanediyl-bis(nitrilomethylidyne)]-bis-phenol schiff-base and its $\mathrm{Mn}$ (II) complex: Synthesis, experimental, and theoretical characterization. J. Struct. Chem. 2013, 54, 1063-1069.

52. Hull, J.F.; Balcells, D.; Sauer, E.L.O.; Raynaud, C.; Brudvig, G.W.; Crabtree, R.H.; Eisenstein, O. Manganese Catalysts for C-H Activation: An Experimental/Theoretical Study Identifies the Stereoelectronic Factor That Controls the Switch between Hydroxylation and Desaturation Pathways. J. Am. Chem. Soc. 2010, 132, 7605-7616.

53. Ji, L.; Franke, A.; Brindell, M.; Oszajca, M.; Zahl, A.; van Eldik, R. Combined Experimental and Theoretical Study on the Reactivity of Compounds I and II in Horseradish Peroxidase Biomimetics. Chem. Eur. J. 2014, 20, 14437-14450.

54. Sun, Y.; Hu, X.; Li, H.; Jalbout, A.F. Metalloporphyrin-Dioxygen Interactions and the Effects of Neutral Axial Ligands. J. Phys. Chem. C 2009, 113, 14316-14323.

55. Balland, V.; Charlot, M.F.; Banse, F.; Girerd, J.J.; Mattioli, T.A.; Bill, E.; Bartoli, J.F.; Battioni, P.; Mansuy, D. Spectroscopic characterization of an Fe-IV intermediate generated by reaction of $\mathrm{XO}^{-}(\mathrm{X}=\mathrm{Cl}, \mathrm{Br})$ with an Fe-II complex bearing a pentadentate non-porphyrinic ligand - Hydroxylation and epoxidation activity. Eur. J. Inorg. Chem. 2004, 2004, 301-308. 
56. Comba, P.; Rajaraman, G. Epoxidation and 1,2-Dihydroxylation of Alkenes by a Nonheme Iron Model System-DFT Supports the Mechanism Proposed by Experiment. Inorg. Chem. 2008, 47, 78-93.

57. Kamachi, T.; Shiota, Y.; Ohta, T.; Yoshizawa, K. Does the Hydroperoxo Species of Cytochrome P450 Participate in Olefin Epoxidation with the Main Oxidant, Compound I? Criticism from Density Functional Theory Calculations. Bull. Chem. Soc. Jpn. 2003, 76, 721-732.

58. Ogliaro, F.; de Visser, S.P.; Cohen, S.; Sharma, P.K.; Shaik, S. Searching for the Second Oxidant in the Catalytic Cycle of Cytochrome P450: A Theoretical Investigation of the Iron(III)-Hydroperoxo Species and Its Epoxidation Pathways. J. Am. Chem. Soc. 2002, 124, 2806-2817.

59. Ostermeier, M.; Limberg, C.; Herwig, C.; Ziemer, B. Stabilizing the Boat Conformation of Piperazines Coordinated to Iron(II): Iso-Butyl Substituents Lead to Robust Oxidation Catalysts via Hyperconjugation. Z. Anorg. Allg. Chem. 2009, 635, 1823-1830.

60. Faponle, A.S.; Quesne, M.G.; Sastri, C.V.; Banse, F.; de Visser, S.P. Differences and Comparisons of the Properties and Reactivities of Iron(III)-hydroperoxo Complexes with Saturated Coordination Sphere. Chem. Eur. J. 2014, 21, 1221-1236.

61. García-Aguilar, J.; Miguel-García, I.; Juan-Juan, J.; Such-Basáñez, I.; Fabián, E.S.; Cazorla-Amorós, D.; Berenguer-Murcia, Á. One step-synthesis of highly dispersed iron species into silica for propylene epoxidation with dioxygen. J. Catal. 2016, 338, 154-167.

62. Quiñonero, D.; Musaev, D.G.; Morokuma, K. Theoretical Studies of the Complex [(BPMEN)Fe(II) $\left(\mathrm{NCCH}_{3}\right)_{2}$ ]$^{2+}$, Precursor of Non-Heme Iron Catalysts for Olefin Epoxidation and Cis-Dihydroxylation. Inorg. Chem. 2003, 42, 8449-8455.

63. Quiñonero, D.; Morokuma, K.; Musaev, D.G.; Mas-Ballesté, R.; Que, L. Metal-Peroxo versus Metal-Oxo Oxidants in Non-Heme Iron-Catalyzed Olefin Oxidations: Computational and Experimental Studies on the Effect of Water. J. Am. Chem. Soc. 2005, 127, 6548-6549.

64. Kwong, H.K.; Lo, P.K.; Lau, K.C.; Lau, T.C. Epoxidation of alkenes and oxidation of alcohols with hydrogen peroxide catalyzed by a manganese(V) nitrido complex. Chem. Commun. 2011, 47, 4273-4275.

65. Miao, C.; Wang, B.; Wang, Y.; Xia, C.; Lee, Y.M.; Nam, W.; Sun, W. Proton-Promoted and Anion-Enhanced Epoxidation of Olefins by Hydrogen Peroxide in the Presence of Nonheme Manganese Catalysts. J. Am. Chem. Soc. 2016, 138, 936-943.

66. Quiñonero, D.; Musaev, D.G.; Morokuma, K. Computational insights to the mechanism of alkene epoxidation by manganese-based catalysts in the presence of bicarbonate. J. Mol. Struct. THEOCHEM 2009, 903, 115-122.

67. Rutkowska-Zbik, D.; Tokarz-Sobieraj, R.; Witko, M. Quantum chemical description of oxygen activation process on Co, Mn, and Mo porphyrins. J. Chem. Theory Comput. 2007, 3, 914-920.

68. Shaik, S.; Hirao, H.; Kumar, D. Reactivity patterns of cytochrome P450 enzymes: Multifunctionality of the active species, and the two states-two oxidants conundrum. Nat. Prod. Rep. 2007, 24, 533-552.

69. Abashkin, Y.G.; Burt, S.K. (Salen)Mn-Catalyzed Epoxidation of Alkenes: A Two-Zone Process with Different Spin-State Channels as Suggested by DFT Study. Org. Lett. 2004, 6, 59-62.

70. Singh, K.K.; Tiwari, M.; Dhar, B.B.; Vanka, K.; Gupta, S.S. Mechanism of Oxygen Atom Transfer from Fe-V(O) to Olefins at Room Temperature. Inorg. Chem. 2015, 54, 6112-6121.

71. Norrby, P.O.; Linde, C.; Aakermark, B. On the Chirality Transfer in the Epoxidation of Alkenes Catalyzed by Mn(salen) Complexes. J. Am. Chem. Soc. 1995, 117, 11035-11036.

72. Linde, C.; Arnold, M.; Åkermark, B.; Norrby, P.O. Is There a Radical Intermediate in the (salen)Mn-Catalyzed Epoxidation of Alkenes? Angew. Chem. Int. Ed. Engl. 1997, 36, 1723-1725.

73. Kürti, L.; Blewett, M.M.; Corey, E.J. Origin of Enantioselectivity in the Jacobsen Epoxidation of Olefins. Org. Lett. 2009, 11, 4592-4595.

74. Linde, C.; Akermark, B.; Norrby, P.; Svensson, M. Timing Is Critical: Effect of Spin Changes on the Diastereoselectivity in Mn(salen)-Catalyzed Epoxidation. J. Am. Chem. Soc. 1999, 121, 5083-5084.

75. Cavallo, L.; Jacobsen, H. Radical Intermediates in the Jacobsen - Katsuki Epoxidation. Angew. Chem. Int. Ed. 2000, 39, 589-592.

76. Cavallo, L.; Jacobsen, H. Manganese-Salen Complexes as Oxygen-Transfer Agents in Catalytic Epoxidations-A Density Functional Study of Mechanistic Aspects. Eur. J. Inorg. Chem. 2003, 2003, 892-902.

77. Jacobsen, H.; Cavallo, L. Donor-Ligand Effect on the Product Distribution in the Manganese-Catalyzed Epoxidation of Olefins: A Computational Assessment. Organometallics 2006, 25, 177-183. 
78. Reiher, M.; Salomon, O.; Artur Hess, B. Reparameterization of hybrid functionals based on energy differences of states of different multiplicity. Theor. Chem. Acc. 2001, 107, 48-55.

79. Rich, J.; Rodríguez, M.; Romero, I.; Fontrodona, X.; van Leeuwen, P.W.N.M.; Freixa, Z.; Sala, X.; Poater, A.; Solà, M. N-Tetradentate SPANamine Derivatives and Their Mn II -Complexes as Catalysts for Epoxidation of Alkenes. Eur. J. Inorg. Chem. 2012, 2013, 1213-1224.

80. Manrique, E.; Poater, A.; Fontrodona, X.; Solà, M.; Rodríguez, M.; Romero, I. Reusable manganese compounds containing pyrazole-based ligands for olefin epoxidation reactions. Dalton Trans. 2015, 44, 17529-17543.

81. Jacobsen, H.; Cavallo, L. A Possible Mechanism for Enantioselectivity in the Chiral Epoxidation of Olefins with [Mn(salen)] Catalysts. Chem. Eur. J. 2001, 8, 800-807.

82. Khavrutskii, I.V.; Musaev, D.G.; Morokuma, K. Epoxidation of unfunctionalized olefins by Mn(salen) catalyst using organic peracids as oxygen source: A theoretical study. Proc. Natl. Acad. Sci. USA 2004, 101, 5743-5748.

83. Malek, K.; Jansen, A.; Li, C.; van Santen, R. Enantioselectivity of immobilized Mn-salen complexes: A computational study. J. Catal. 2007, 246, 127-135.

84. Malek, K.; Li, C.; van Santen, R.A. New theoretical insights into epoxidation of alkenes by immobilized Mn-salen complexes in mesopores: Effects of substrate, linker and confinement. J. Mol. Catal. A Chem. 2007, 271, 98-104.

85. Zabrodsky, H.; Avnir, D. Chirality Continuous Symmetry Measures. 4. Chirality. J. Am. Chem. Soc. 1995, $117,462-473$.

86. Oxford, G.A.E.; Snurr, R.Q.; Broadbelt, L.J. Hybrid Quantum Mechanics/Molecular Mechanics Investigation of (salen)Mn for use in Metal-Organic Frameworks. Ind. Eng. Chem. Res. 2010, 49, 10965-10973.

87. Oxford, G.A.E.; Dubbeldam, D.; Broadbelt, L.J.; Snurr, R. Elucidating steric effects on enantioselective epoxidation catalyzed by (salen)Mn in metal-organic frameworks. J. Mol. Catal. A Chem. 2011, 334, 89-97.

88. Sainna, M.A.; Kumar, S.; Kumar, D.; Fornarini, S.; Crestoni, M.E.; de Visser, S.P. A comprehensive test set of epoxidation rate constants for iron(IV)-oxo porphyrin cation radical complexes. Chem. Sci. 2015, $6,1516-1529$.

89. Yi, W.; Yuan, L.; Kun, Y.; Zhengwen, H.; Jing, T.; Xu, F.; Hong, G.; Yong, W. What factors influence the reactivity of $\mathrm{C}-\mathrm{H}$ hydroxylation and $\mathrm{C}=\mathrm{C}$ epoxidation by $[$ Fe-IV(L-ax)(1,4,8,11-tetramethyl-1,4,8,11-tetraazacyclotetradecane $)(\mathrm{O})]^{n+}$. J. Biol. Inorg. Chem. 2015, 20, 1123-1134.

90. Kaczmarzyk, T.; Dziedzic-Kocurek, K.; Rutkowska, I.; Dziliński, K. Mössbauer study of a tetrakis (pentafluorophenyl) porphyrin iron (III) chloride in comparison with the fluorine unsubstituted analogue. Nukleonika 2015, 60, 57-61.

91. De Visser, S.P. Predictive Studies of Oxygen Atom Transfer Reactions by Compound I of Cytochrome P450: Aliphatic and Aromatic Hydroxylation, Epoxidation, and Sulfoxidation. In Advances in Inorganic Chemistry Volume 64: Inorganic Bioinorganic Reaction Mechanisms; Advances in Inorganic Chemistry; VanEldik, R., Ed.; Elsevier BV: Amsterdam, The Netherlands, 2012; Volume 64, pp. 1-31.

92. Shaik, S.; de Visser, S.P.; Kumar, D. One oxidant, many pathways: A theoretical perspective of monooxygenation mechanisms by cytochrome P450 enzymes. J. Biol. Inorg. Chem. 2004, 9, 661-668.

93. Shaik, S.; Woggon, W.D.; Kozuch, S.; Leifels, T.; Meyer, D.; Sbaragli, L. New Synthetic Models of Cytochrome P450: How Different Are They from the Natural Species? Synlett 2005, 675-684.

94. Shaik, S.; Lai, W.; Chen, H.; Wang, Y. The Valence Bond Way: Reactivity Patterns of Cytochrome P450 Enzymes and Synthetic Analogs. Acc. Chem. Res. 2010, 43, 1154-1165.

95. Kumar, D.; de Visser, S.P.; Sharma, P.K.; Derat, E.; Shaik, S. The intrinsic axial ligand effect on propene oxidation by horseradish peroxidase versus cytochrome P450 enzymes. J. Biol. Inorg. Chem. 2005, 10, 181-189.

96. Zhang, J.; Ji, L.; Liu, W. In Silico Prediction of Cytochrome P450-Mediated Biotransformations of Xenobiotics: A Case Study of Epoxidation. Chem. Res. Toxicol. 2015, 28, 1522-1531.

97. Drzewiecka-Matuszek, A.; Rutkowska-Zbik, D.; Witko, M. Hydrogen peroxide as oxidant in bio-mimetic catalysis by manganese porphyrin: Theoretical DFT studies. Can. J. Chem. 2013, 91, 642-647.

98. Teixeira, F.; Melo, A.; Cordeiro, M.N.D.S. Calibration sets and the accuracy of vibrational scaling factors: A case study with the X3LYP hybrid functional. J. Chem. Phys. 2010, 133, 114109, doi:10.1063/1.3493630. 
99. Wistuba, T.; Limberg, C. The Reaction of Permanganyl Chloride with Olefins: Intermediates and Mechanism as Derived from Matrix-Isolation Studies and Density Functional Theory Calculations. Chem. Eur. J. 2001, 7, 4674-4685.

100. Srour, H.; Maux, P.L.; Chevance, S.; Simonneaux, G. Metal-catalyzed asymmetric sulfoxidation, epoxidation and hydroxylation by hydrogen peroxide. Coord. Chem. Rev. 2013, 257, 3030-3050.

101. Xia, Q.H.; Ge, H.Q.; Ye, C.P.; Liu, Z.M.; Su, K.X. Advances in Homogeneous and Heterogeneous Catalytic Asymmetric Epoxidation. Chem. Rev. 2005, 105, 1603-1662.

102. Brandt, P.; Norrby, P.; Daly, A.M.; Gilheany, D.G. Chromium-Salen-Mediated Alkene Epoxidation: A Theoretical and Experimental Study Indicates the Importance of Spin-Surface Crossing and the Presence of a Discrete Intermediate. Chem. Eur. J. 2002, 8, 4299-4307.

103. Venkataramanan, N.S.; Rajagopal, S.; Suvitha, A.; Kawazoe, Y. A combined experimental and theoretical investigation on the oxygenation of organic sulfides by oxo(salen)chromium(V) ion. J. Phys. Org. Chem. 2009, 22, 650-660.

104. Vandichel, M.; Leus, K.; der Voort, P.V.; Waroquier, M.; Speybroeck, V.V. Mechanistic insight into the cyclohexene epoxidation with $\mathrm{VO}(\mathrm{acac})_{2}$ and tert-butyl hydroperoxide. J. Catal. 2012, 294, 1-18.

105. Adão, P.; Pessoa, J.C.; Henriques, R.T.; Kuznetov, M.L.; Avecilla, F.; Maurya, M.R.; Kumar, U.; Correia, I. Synthesis, Characterization, and Application of Vanadium-Salan Complexes in Oxygen Transfer Reactions. Inorg. Chem. 2009, 48, 3542-3561.

106. Kirillova, M.V.; Kuznetov, M.L.; Romakh, V.B.; Shul'pina, L.S.; Silva, J.D.; Pombeiro, A.J.L.; Shul'pin, G.B. Mechanism of oxidations with $\mathrm{H}_{2} \mathrm{O}_{2}$ catalyzed by vanadate anion or oxovanadium(V) triethanolaminate (vanadatrane) in combination with pyrazine-2-carboxylic acid (PCA): Kinetic and DFT studies. J. Catal. 2009, 267, 140-157.

107. Kuznetov, M.L.; Pessoa, J.C. Epoxidation of olefins catalyzed by vanadium-salan complexes: A theoretical mechanistic study. J. Chem. Soc. Dalton Trans. 2009, 2009, 5460-5468.

108. Leus, K.; Muylaert, I.; Vandichel, M.; Marin, G.B.; Waroquier, M.; Van Speybroeck, V.; Van Der Voort, P. The remarkable catalytic activity of the saturated metal organic framework V-MIL-47 in the cyclohexene oxidation. Chem. Commun. 2010, 46, 5085-5087.

109. Aschi, M.; Crucianelli, M.; Giuseppe, A.D.; Nicola, C.D.; Marchetti, F. Insights on the mechanistic features of catalytic oxidations of simple and conjugated olefins promoted by $\mathrm{VO}(\mathrm{acac})_{2} / \mathrm{H}_{2} \mathrm{O}_{2}$ system, in acetonitrile: A computational study. Catal. Today 2012, 192, 56-62.

110. Tahmasebi, V.; Grivani, G.; Bruno, G. Synthesis, characterization, crystal structure determination and catalytic activity in epoxidation reaction of two new oxidovanadium(IV) Schiff base complexes. J. Mol. Struct. 2016, 1123, 367-374.

111. Sever, R.R.; Root, T.W. DFT Study of Solvent Coordination Effects on Titanium-Based Epoxidation Catalysts. Part Two: Reactivity of Titanium Hydroperoxo Complexes in Ethylene Epoxidation. J. Phys. Chem. B 2003, 107, 4090-4099.

112. Limtrakul, J.; Inntam, C.; Truong, T.N. Density functional theory study of the ethylene epoxidation over Ti-substituted silicalite (TS-1). J. Mol. Catal. A Chem. 2004, 207, 139-148.

113. Kholdeeva, O.A. Hydrogen Peroxide Activation over Ti IV: What Have We Learned from Studies on Ti-Containing Polyoxometalates? Eur. J. Inorg. Chem. 2013, 2013, 1595-1605.

114. Antonova, N.S.; Carbo, J.J.; Kortz, U.; Kholdeeva, O.A.; Poblet, J.M. Mechanistic Insights into Alkene Epoxidation with $\mathrm{H}_{2} \mathrm{O}_{2}$ by Ti- and other TM-Containing Polyoxometalates: Role of the Metal Nature and Coordination Environment. J. Am. Chem. Soc. 2010, 132, 7488-7497.

115. Costa, P.J.; Calhorda, M.J.; Kuhn, F.E. Olefin Epoxidation Catalyzed by eta5-Cyclopentadienyl Molybdenum Compounds: A Computational Study. Organometallics 2010, 29, 303-311.

116. Schmidt, A.; Grover, N.; Zimmermann, T.K.; Graser, L.; Cokoja, M.; Pöthig, A.; Kühn, F.E. Synthesis and characterization of novel cyclopentadienyl molybdenum imidazo[1,5-a]pyridine-3-ylidene complexes and their application in olefin epoxidation catalysis. J. Catal. 2014, 319, 119-126.

117. Drees, M.; Hauser, S.A.; Cokoja, M.; Kühn, F.E. DFT studies on the reaction pathway of the catalytic olefin epoxidation with $\mathrm{CpMoCF}_{3}$ dioxo and oxo-peroxo complexes. J. Organomet. Chem. 2013, 748, 36-45.

118. Morlot, J.; Uyttebroeck, N.; Agustin, D.; Poli, R. Solvent-Free Epoxidation of Olefins Catalyzed by $\mathrm{MoO}_{2}$ (SAP): A New Mode of tert-Butylhydroperoxide Activation. ChemCatChem 2012, 5, 601-611. 
119. Poater, A.; Falivene, L.; Cavallo, L.; Llobet, A.; Rodríguez, M.; Romero, I.; Solà, M. Simple and cheap steric and electronic characterization of the reactivity of $\mathrm{Ru}(\mathrm{II})$ complexes containing oxazoline ligands as epoxidation catalysts. Chem. Phys. Lett. 2013, 577, 142-146.

120. Coquet, R.; Tada, M.; Iwasawa, Y. Energy-gaining formation and catalytic behavior of active structures in a $\mathrm{SiO}_{2}$-supported unsaturated $\mathrm{Ru}$ complex catalyst for alkene epoxidation by DFT calculations. Phys. Chem. Chem. Phys. 2007, 9, 6040-6046.

121. Aguiló, J.; Francàs, L.; Bofill, R.; Gil-Sepulcre, M.; García-Antón, J.; Poater, A.; Llobet, A.; Escriche, L.; Meyer, F.; Sala, X. Powerful Bis-facially Pyrazolate-Bridged Dinuclear Ruthenium Epoxidation Catalyst. Inorg. Chem. 2015, 54, 6782-6791.

122. Di Giovanni, C.; Poater, A.; Benet-Buchholz, J.; Cavallo, L.; Solà, M.; Llobet, A. Dinuclear Ru-Aqua Complexes for Selective Epoxidation Catalysis Based on Supramolecular Substrate Orientation Effects. Chem. Eur. J. 2014, 20, 3898-3902.

123. Cavazzini, M.; Quici, S.; Pozzi, G. Hydrolytic kinetic resolution of terminal epoxides catalyzed by fluorous chiral Co(salen) complexes. Tetrahedron 2002, 58, 3943-3949.

124. Grivani, G.; Vakili, M.; Khalaji, A.D.; Bruno, G.; Rudbari, H.A.; Taghavi, M.; Tahmasebi, V. Synthesis, characterization, crystal structure determination, computational study, and thermal decomposition into $\mathrm{NiO}$ nano-particles of a new NiIIL2 Schiff base complex ( $\mathrm{L}=2-\{(\mathrm{E})-[2$-chloroethyl)imino]methylphenolate). J. Mol. Struct. 2014, 1072, 77-83.

125. Düzenli, D.; Atmaca, D.O.; Gezer, M.G.; Onal, I. A density functional theory study of partial oxidation of propylene on $\mathrm{Cu}_{2} \mathrm{O}(001)$ and $\mathrm{CuO}(001)$ surfaces. Appl. Surf. Sci. 2015, 355, 660-666.

126. Salman, A.W.; Rehman, G.U.; Abdullah, N.; Budagumpi, S.; Endud, S.; Abdallah, H.H. Synthesis, characterization, density function theory and catalytic performances of palladium(II)- $N$-heterocyclic carbene complexes derived from benzimidazol-2-ylidenes. Inorg. Chim. Acta 2015, 438, 14-22.

127. Salman, A.W.; Rehman, G.U.; Abdullah, N.; Budagumpi, S.; Endud, S.; Abdallah, H.H.; Wong, W.Y. Sterically modulated palladium(II)-N-heterocyclic carbene complexes for the catalytic oxidation of olefins: Synthesis, crystal structure, characterization and DFT studies. Polyhedron 2014, 81, 499-510.

128. Dellamorte, J.; Lauterbach, J.; Barteau, M. Palladium-silver bimetallic catalysts with improved activity and selectivity for ethylene epoxidation. Appl. Catal. A 2011, 391, 281-288.

129. Robinson, J.R.; Yadav, J.; Fan, X.; Stanton, G.R.; Schelter, E.J.; Pericàs, M.A.; Walsh, P.J. Non-Covalent Immobilization of Rare Earth Heterobimetallic Frameworks and their Reactivity in an Asymmetric Michael Addition. Adv. Synth. Catal. 2014, 356, 1243-1254.

130. Yang, B.; Manz, T.A. Hafnium catalysts for direct alkene epoxidation using molecular oxygen as oxidant. RSC Adv. 2015, 5, 12311-12322.

131. Yan, W.; Ramanathan, A.; Ghanta, M.; Subramaniam, B. Towards highly selective ethylene epoxidation catalysts using hydrogen peroxide and tungsten- or niobium-incorporated mesoporous silicate (KIT-6). Catal. Sci. Technol. 2014, 4, 4433-4439.

132. Abashkin, Y.G.; Burt, S.K. (salen)Mn III Compounds as Nonpeptidyl Mimics of Catalase. Mechanism-Based Tuning of Catalase Activity: A Theoretical Study. Inorg. Chem. 2005, 44, 1425-1432.

133. Hirao, H.; Kumar, D.; Thiel, W.; Shaik, S. Two States and Two More in the Mechanisms of Hydroxylation and Epoxidation by Cytochrome P450. J. Am. Chem. Soc. 2005, 127, 13007-13018.

134. De Visser, S.; Stillman, M. Challenging Density Functional Theory Calculations with Hemes and Porphyrins. Int. J. Mol. Sci. 2016, 17, 519, doi:10.3390/ijms17040519.

135. Teixeira, F.; Mosquera, R.A.; Melo, A.; Freire, C.; Cordeiro, M.N.D.S. Effects of Axial Coordination on Immobilized Mn(salen) Catalysts. J. Phys. Chem. A 2014, 118, 10788-10796.

136. Curtiss, L.A.; Redfern, P.C.; Raghavachari, K.; Rassolov, V.; Pople, J.A. Gaussian-3 theory using reduced Moller-Plesset order. J. Chem. Phys. 1999, 110, 4703-4709.

137. Ufimtsev, I.S.; Martiínez, T.J. Graphical Processing Units for Quantum Chemistry. Comput. Sci. Eng. 2008, 10, 26-34.

138. Titov, A.V.; Ufimtsev, I.S.; Luehr, N.; Martsíinez, T.J. Generating Efficient Quantum Chemistry Codes for Novel Architectures. J. Chem. Theor. Comput. 2013, 9, 213-221.

139. Song, C.; Martínez, T.J. Atomic orbital-based SOS-MP2 with tensor hypercontraction. I. GPU-based tensor construction and exploiting sparsity. J. Chem. Phys. 2016, 144, 174111, doi:10.1063/1.4948438. 
140. Fales, B.S.; Levine, B.G. Nanoscale Multireference Quantum Chemistry: Full Configuration Interaction on Graphical Processing Units. J. Chem. Theor. Comput. 2015, 11, 4708-4716.

141. Stratmann, R.; Scuseria, G.E.; Frisch, M.J. Achieving linear scaling in exchange-correlation density functional quadratures. Chem. Phys. Lett. 1996, 257, 213-223.

142. Izsák, R.; Neese, F. An overlap fitted chain of spheres exchange method. J. Chem. Phys. 2011, 135, 144105, doi:10.1063/1.3646921.

143. Van Dam, H.J.J.; Vishnu, A.; de Jong, W.A. A Case for Soft Error Detection and Correction in Computational Chemistry. J. Chem. Theor. Comput. 2013, 9, 3995-4005.

(C) 2016 by the authors; licensee MDPI, Basel, Switzerland. This article is an open access article distributed under the terms and conditions of the Creative Commons Attribution (CC-BY) license (http:/ / creativecommons.org/licenses/by/4.0/). 